\title{
Dynamic Linear Panel Regression Models with Interactive Fixed Effects*
}

\author{
Hyungsik Roger Moon Martin Weidner $^{\ddagger}$
}

August 10, 2015

*This paper is based on an unpublished manuscript of the authors that was circulated under the title "Likelihood Expansion for Panel Regression Models with Factors" but is now completely assimilated by the current paper and Moon and Weidner (2015). We greatly appreciate comments from the participants in the Far Eastern Meeting of the Econometric Society 2008, the SITE 2008 Conference, the All-UC-Econometrics Conference 2008, the July 2008 Conference in Honour of Peter Phillips in Singapore, the International Panel Data Conference 2009, the North American Summer Meeting of the Econometric Society 2009, and from seminar participants at Penn State, UCLA, and USC. We are also grateful for the comments and suggestions of Guido Kuersteiner, Peter Phillips, and anonymous referees. Moon is grateful for the financial support from the NSF via grant SES 0920903 and the faculty development award from USC. Weidner acknowledges support from the Economic and Social Research Council through the ESRC Centre for Microdata Methods and Practice grant RES-589-28-0001.

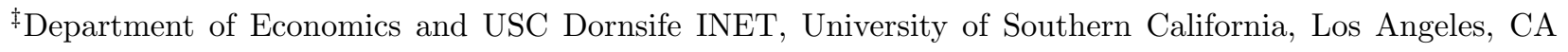
90089-0253. Email: moonr@usc.edu. Department of Economics, Yonsei University, Seoul, Korea.

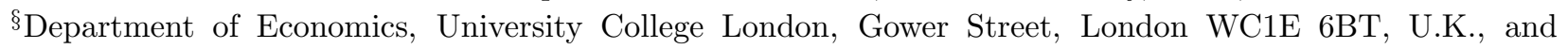
CeMMaP. Email: m.weidner@ucl.ac.uk. 


\begin{abstract}
We analyze linear panel regression models with interactive fixed effects and predetermined regressors, for example lagged-dependent variables. The first-order asymptotic theory of the least squares (LS) estimator of the regression coefficients is worked out in the limit where both the cross-sectional dimension and the number of time periods become large. We find two sources of asymptotic bias of the LS estimator: bias due to correlation or heteroscedasticity of the idiosyncratic error term, and bias due to predetermined (as opposed to strictly exogenous) regressors. We provide a bias-corrected LS estimator. We also present bias-corrected versions of the three classical test statistics (Wald, LR, and LM test) and show their asymptotic distribution is a $\chi^{2}$-distribution. Monte Carlo simulations show the bias correction of the LS estimator and of the test statistics also work well for finite sample sizes.
\end{abstract}




\section{Dynamic Panel With Interactive Effects}

\section{Introduction}

In this paper, we study a linear panel regression model in which the individual fixed effects $\lambda_{i}$, called factor loadings, interact with common time-specific effects $f_{t}$, called factors. This interactive fixed effect specification contains the conventional individual specific effects and timespecific effects as special cases but is significantly more flexible because it allows the factors $f_{t}$ to affect each individual with a different loading $\lambda_{i}$.

Factor models have been widely studied in various economics disciplines, for example, in asset pricing, forecasting, empirical macro, and empirical labor economics. ${ }^{1}$ The panel literature often uses factor models to represent time-varying individual effects (or heterogenous time effects), so-called interactive fixed effects. For panels with a large cross-sectional dimension $(N)$ but a short time dimension (T), Holtz-Eakin, Newey, and Rosen (1988) (hereafter HNR) study a linear panel regression model with interactive fixed effects and lagged dependent variables. To solve the incidental parameter problem caused by the $\lambda_{i}$ 's, they estimate a quasi-differenced version of the model using appropriate lagged variables as instruments, and treating $f_{t}$ 's as a fixed number of parameters to estimate. Ahn, Lee, and Schmidt (2001) also consider large $N$ but short $T$ panels. Instead of eliminating the individual effects $\lambda_{i}$ by transforming the panel data, they impose various second-moment restrictions including the correlated random effects $\lambda_{i}$, and derive moment conditions to estimate the regression coefficients. The more recent literature considers panels with comparable size of $N$ and $T$. The interactive fixed effect panel regression model of Pesaran (2006) allows heterogenous regression coefficients. Pesaran's estimator is the common correlated effect (CCE) estimator that uses the cross-sectional averages of the dependent variable and the independent variables as control functions for the interactive fixed effects. ${ }^{2}$

Among the interactive fixed effect panel literature, most closely related to our paper is Bai (2009). Bai assumes the regressors are strictly exogenous and the number of factors is known. The estimator he investigates is the least squares (LS) estimator, which minimizes the sum of squared residuals of the model jointly over the regression coefficients and the fixed effect parameters $\lambda_{i}$ and $f_{t}{ }^{3}$ Using alternative asymptotics where $N, T \rightarrow \infty$ at the same rate, ${ }^{4}$ Bai shows the LS estimator is $\sqrt{N T}$-consistent and asymptotically normal, but may have an asymptotic bias. The bias in the normal limiting distribution occurs when the regression errors are correlated or heteroscedastic. Bai also shows how to estimate the bias, and proposes a bias-corrected estimator.

Following the methodology in Bai (2009), we investigate the LS estimator for a linear panel 


\section{Dynamic Panel With Interactive Effects}

regression with a known number of interactive fixed effects. The main difference from Bai is that we consider predetermined regressors, thus allowing feedback of past outcomes to future regressors. One of the main findings of the present paper is that the limit distribution of the LS

estimator has two types of biases: one type of bias due to correlated or heteroscedastic errors (the same bias as in Bai) and the other type of bias due to the predetermined regressors. This additional bias term is analogous to the incidental parameter bias of Nickell (1981) in finite $T$ and the bias in Hahn and Kuersteiner (2002) in large $T$.

In addition to allowing for predetermined regressors, we also extend Bai's results to models in which both "low-rank regressors" (e.g., time-invariant and common regressors, or interactions of those two) and "high-rank-regressors" (almost all other regressors that vary across individuals and over time) are present simultaneously, wheras Bai (2009) only considers the low-rank regressors separately and in a restrictive setting (in particular, not allowing for regressors that are obtained by interacting time-invariant and common variables). A general treatment of lowrank regressors is desirable because they often occur in applied work, for example, Gobillon and Magnac (2013). The analysis of those regressors is challenging, however, because the unobserved interactive fixed effects also represent a low-rank $N \times T$ matrix, thus posing a non-trivial identification problem for low-rank regressors, which needs to be addressed. We provide conditions under which the different types of regressors are identified jointly, and under which they can be estimated consistently as $N$ and $T$ grow large.

Another contribution of this paper is to establish the asymptotic theory of the three classical test statistics (Wald test, LR test, and LM (or score) test) for testing restrictions on the regression coefficients in a large $N, T$ panel framework. ${ }^{5}$ Regarding testing for coefficient restrictions, Bai (2009) investigates the Wald test based on the bias-corrected LS estimator, and HNR consider the LR test in their 2SLS estimation framework with fixed $T .{ }^{6}$ What we show is that the conventional LR and LM test statistics based on the LS profile objective function have non-central chi-square limits due to incidental parameters in the interactive fixed effects. We therefore propose modified LR and LM tests whose asymptotic distributions are conventional chi-square distributions.

To establish the asymptotic theories of the LS estimator and the three classical tests, we use the quadratic approximation of the profile LS objective function derived in Moon and Weidner (2015). This method is different from Bai (2009), who uses the first-order condition of the LS optimization problem as the starting point of his analysis. One advantage of our methodology is that it can also directly be applied to derive the asymptotic properties of the LR and LM test statistics. 


\section{Dynamic Panel With Interactive Effects}

In this paper, we assume the regressors are not endogenous and the number of factors is known, which might be restrictive in some applications. In other papers, we study how to relax these restrictions. Moon and Weidner (2015) investigates the asymptotic properties of the LS estimator of the linear panel regression model with factors when the number of factors is unknown and extra factors are included unnecessarily in the estimation. We find that under suitable conditions, ${ }^{7}$ the limit distribution of the LS estimator is unchanged when the number of factors is overestimated. The extension to allow for endogenous regressors is very briefly discussed in section 6 of the current paper, and is closely related to the results in Moon, Shum, and Weidner (2012) (hereafter MSW). MSW's main purpose is to extend the random coefficient multinomial logit demand model (known as the BLP demand model from Berry, Levinsohn, and Pakes (1995)) by allowing for interactive product and market specific fixed effects. Although the main model of interest is quite different from the linear panel regression model of the current paper, MSW's econometrics framework is directly applicable to the model of the current paper with endogenous regressors. ${ }^{8}$

Comparing the different estimation approaches for interactive fixed effect panel regressions proposed in the literature, it seems fair to say that the LS estimator in Bai (2009) and our paper, the CCE estimator of Pesaran (2006), and the IV estimator based on quasi-differencing in HNR all have their own relative advantages and disadvantages. These three estimation methods handle the interactive fixed effects quite differently. The LS method concentrates out the interactive fixed effects by taking out the principal components. The CCE method controls the factor (or time effects) using the cross-sectional averages of the dependent and independent variables. The HNR's approach quasi-differences out the individual effects, treating the remaining time effects as parameters to estimate. The IV estimator of HNR should work well when $T$ is short, but is expected to also suffer from an incidental parameter problem when $T$ becomes large, because then many factors need to be estimated as parameters that enter the model non-linearly. Pesaran's CCE estimation method does not require the number of factors to be known and does not require the strong factor assumption that we will impose below, but for the CCE estimator to work, not only the DGPs of the dependent variable (e.g., the regression model) but also the DGPs of the explanatory variables need to be restricted such that their cross-sectional average can control for unobserved factors. The LS estimator and its bias-corrected version perform well under relatively weak restrictions on the regressors, but requires that $T$ should not be too small and that the factors should be sufficiently strong to be correctly picked up as the leading principal components.

The paper is organized as follows. In section 2, we introduce the interactive fixed effect model 


\section{Dynamic Panel With Interactive Effects}

and provide conditions for identifying the regression coefficients in the presence of the interactive fixed effects. In section 3, we define the LS estimator of the regression parameters and provide a set of assumptions that are sufficient to show consistency of the LS estimator. In section 4 , we work out the asymptotic distribution of the LS estimator under alternative asymptotics. We also provide a consistent estimator for the asymptotic bias and a bias-corrected LS estimator. In section 5, we consider the Wald, LR, and LM tests for testing restrictions on the regression coefficients of the model. We present bias-corrected versions of these tests and show that they have chi-square limiting distributions. In section 6 , we briefly discuss how to estimate the interactive fixed effect linear panel regression when the regressors are endogenous. In section 7 , we present Monte Carlo simulation results for an $\mathrm{AR}(1)$ model with interactive fixed effects. The simulations show the LS estimator for the AR(1) coefficient is biased, and the tests based on it can have severe size distortions and power asymmetries, wheras the bias-corrected LS estimator and test statistics have better properties. We conclude in section 8. We present all proofs of theorems and some technical details in the appendix or supplementary material.

A few words on notation are due. For a column vector $v$, the Euclidean norm is defined by $\|v\|=\sqrt{v^{\prime} v}$. For the $n$-th largest eigenvalues (counting multiple eigenvalues multiple times) of a symmetric matrix $B$, we write $\mu_{n}(B)$. For an $m \times n$ matrix $A$, the Frobenius norm is $\|A\|_{F}=$

$\sqrt{\operatorname{Tr}\left(A A^{\prime}\right)}$, and the spectral norm is $\|A\|=\max _{0 \neq v \in \mathbb{R}^{n}} \frac{\|A v\|}{\|v\|}$, or equivalently $\|A\|=\sqrt{\mu_{1}\left(A^{\prime} A\right)}$. Furthermore, we define $P_{A}=A\left(A^{\prime} A\right)^{\dagger} A^{\prime}$ and $M_{A}=\mathbb{I}-A\left(A^{\prime} A\right)^{\dagger} A^{\prime}$, where $\mathbb{I}$ is the $m \times m$ identity matrix, and $\left(A^{\prime} A\right)^{\dagger}$ is the Moore-Penrose pseudoinverse, to allow for the case that $A$ is not of full column rank. For square matrices $B, C$, we write $B>C$ (or $B \geq C$ ) to indicate $B-C$ is positive (semi) definite. For a positive definite symmetric matrix $A$, we write $A^{1 / 2}$ and $A^{-1 / 2}$ for the unique symmetric matrices that satisfy $A^{1 / 2} A^{1 / 2}=A$ and $A^{-1 / 2} A^{-1 / 2}=A^{-1}$. We use $\nabla$ for the gradient of a function; that is, $\nabla f(x)$ is the column vector of partial derivatives of $f$ with respect to each component of $x$. We use "wpa1" for "with probability approaching one".

\section{Model and Identification}

We study the following panel regression model with cross-sectional size $N$, and $T$ time periods:

$$
Y_{i t}=\beta^{0 \prime} X_{i t}+\lambda_{i}^{0 \prime} f_{t}^{0}+e_{i t}, \quad i=1 \ldots N, \quad t=1 \ldots T,
$$

where $X_{i t}$ is a $K \times 1$ vector of observable regressors, $\beta^{0}$ is a $K \times 1$ vector of regression coefficients, $\lambda_{i}^{0}$ is an $R \times 1$ vector of unobserved factor loadings, $f_{t}^{0}$ is an $R \times 1$ vector of unobserved common factors, and $e_{i t}$ are unobserved errors. The superscript zero indicates the true parameters. We 


\section{Dynamic Panel With Interactive Effects}

write $f_{t r}^{0}$ and $\lambda_{i r}^{0}$, where $r=1, \ldots, R$, for the components of $\lambda_{i}^{0}$ and $f_{t}^{0}$, respectively. $R$ is the number of factors. Note that we can have $f_{t r}^{0}=1$ for all $t$ and a particular $r$, in which case the corresponding $\lambda_{i r}^{0}$ become standard individual-specific effects. Analogously, we can have $\lambda_{i r}^{0}=1$ for all $i$ and a particular $r$, so that the corresponding $f_{t r}^{0}$ become standard time-specific effects.

Throughout this paper, we assume the true number of factors $R$ is known. ${ }^{9}$ We introduce the notation $\beta^{0} \cdot X=\sum_{k=1}^{K} \beta_{k}^{0} X_{k}$. In matrix notation, the model can then be written as

$$
Y=\beta^{0} \cdot X+\lambda^{0} f^{0 \prime}+e
$$

where $Y, X_{k}$, and $e$ are $N \times T$ matrices, $\lambda^{0}$ is an $N \times R$ matrix, and $f^{0}$ is a $T \times R$ matrix. The elements of $X_{k}$ are denoted by $X_{k, i t}$.

We separate the $K$ regressors into $K_{1}$ "low-rank regressors" $X_{l}, l=1, \ldots, K_{1}$, and $K_{2}=$ $K-K_{1}$ "high-rank regressors" $X_{m}, m=K_{1}+1, \ldots, K$. Each low-rank regressor $l=1, \ldots, L$ is assumed to satisfy $\operatorname{rank}\left(X_{l}\right)=1$. Therefore, we can write $X_{l}=w_{l} v_{l}^{\prime}$, where $w_{l}$ is an $N$-vector and $v_{l}$ is a $T$-vector, and we also define the $N \times K_{1}$ matrix $w=\left(w_{1}, \ldots, w_{K_{1}}\right)$ and the $T \times K_{1}$ matrix $v=\left(v_{1}, \ldots, v_{K_{1}}\right)$.

Let $l=1, \ldots, K_{1}$. The two most prominent types of low-rank regressors are time-invariant regressors, which satisfy $X_{l, i t}=Z_{i}$ for all $i, t$, and common (or cross-sectionally invariant) regressors, in which case $X_{l, i t}=W_{t}$ for all $i, t$. Here, $Z_{i}$ and $W_{t}$ are some observed variables, which only vary over $i$ or $t$, respectively. A more general low-rank regressor can be obtained by interacting $Z_{i}$ and $W_{t}$ multiplicatively, namely, $X_{l, i t}=Z_{i} W_{t}$, an empirical example of which is given in Gobillon and Magnac (2013). In these examples, and probably for the vast majority of applications, the low-rank regressors all satisfy $\operatorname{rank}\left(X_{l}\right)=1$, but our results can easily be extended to more general low-rank regressors. ${ }^{10}$

High-rank regressors are those whose distribution guarantees they have high rank (usually full rank) when considered as an $N \times T$ matrix. For example, a regressor whose entries satisfy $X_{m, i t} \sim \operatorname{iid} \mathcal{N}(\mu, \sigma)$, with $\mu \in \mathbb{R}$ and $\sigma>0$, satisfies $\operatorname{rank}\left(X_{m}\right)=\min (N, T)$ with probability one.

This separation of the regressors into low- and high-rank regressors is important to formulate our assumptions for identification and consistency, but actually plays no role in the estimation 
and inference procedures for $\widehat{\beta}$ discussed below.

\section{Assumption ID (Assumptions for Identification).}

(i) Existence of Second Moments:

The second moments of $X_{k, i t}$ and $e_{i t}$ conditional on $\lambda^{0}, f^{0}, w$ exist for all $i, t, k$.

(ii) Mean Zero Errors and Exogeneity:

$\mathbb{E}\left(e_{i t} \mid \lambda^{0}, f^{0}, w\right)=0$, and $\mathbb{E}\left(X_{k, i t} e_{i t} \mid \lambda^{0}, f^{0}, w\right)=0, \quad$ a.s., for all $i, t, k$.

The following two assumptions only need to be imposed if $K_{1}>0$, that is, if low-rank regressors are present:

\section{(iii) Non-collinearity of Low-Rank Regressors:}

Consider linear combinations $\alpha \cdot X_{\text {low }}=\sum_{l=1}^{K_{1}} \alpha_{l} X_{l}$ of the low-rank regressors $X_{l}$ with $\alpha \in \mathbb{R}^{K_{1}}$. For all $\alpha \neq 0$, we assume

$$
\mathbb{E}\left[\left(\alpha \cdot X_{\text {low }}\right) M_{f^{0}}\left(\alpha \cdot X_{\text {low }}\right)^{\prime} \mid \lambda^{0}, f^{0}, w\right] \neq 0, \quad \text { a.s. }
$$

(iv) No Collinearity between Factor Loadings and Low-Rank Regressors: $\operatorname{rank}\left(M_{w} \lambda^{0}\right)=\operatorname{rank}\left(\lambda^{0}\right){ }^{11}$

The following assumption only needs to be imposed if $K_{2}>0$, that is, if high-rank regressors are present:

(v) Non-collinearity of High-Rank Regressors:

Consider linear combinations $\alpha \cdot X_{\text {high }}=\sum_{m=K_{1}+1}^{K} \alpha_{m} X_{m}$ of the high-rank regressors $X_{m}$ for $\alpha \in \mathbb{R}^{K_{2}}$, where the components of the $K_{2}$-vector $\alpha$ are denoted by $\alpha_{K_{1}+1}$ to $\alpha_{K}$. For all $\alpha \neq 0$, we assume

$$
\operatorname{rank}\left\{\mathbb{E}\left[\left(\alpha \cdot X_{\text {high }}\right)\left(\alpha \cdot X_{\text {high }}\right)^{\prime} \mid \lambda^{0}, f^{0}, w\right]\right\}>2 R+K_{1}, \quad \text { a.s. }
$$

All expectations in the assumptions are conditional on $\lambda^{0}, f^{0}$, and $w$; in particular, $e_{i t}$ is not allowed to be correlated with $\lambda^{0}, f^{0}$, and $w$. However, $e_{i t}$ is allowed to be correlated with $v$ (i.e., predetermined low-rank regressors are allowed). If desired, one can interchange the role of $N$ and $T$ in the assumptions, by using the formal symmetry of the model under exchange of the panel dimensions $\left(N \leftrightarrow T, \lambda^{0} \leftrightarrow f^{0}, Y \leftrightarrow Y^{\prime}, X_{k} \leftrightarrow X_{k}^{\prime}, w \leftrightarrow v\right)$.

Assumptions ID $(i)$ and $(i i)$ have standard interpretations, but the other assumptions require some further discussion. 


\section{Dynamic Panel With Interactive Effects}

Assumption ID $($ iii $)$ states the low-rank regressors are non-collinear even after projecting out all variation that is explained by the true factors $f^{0}$. This assumption would, for example, be violated if $v_{l}=f_{r}^{0}$ for some $l=1, \ldots, K_{1}$ and $r=1, \ldots, R$, because then $X_{l} M_{f^{0}}=0$ and we can choose $\alpha$ such that $X_{\text {low }}=X_{l}$. Similarly, Assumption ID $(i v)$ rules out, for example, that $w_{l}=\lambda_{r}^{0}$ for some $l=1, \ldots, K_{1}$ and $r=1, \ldots, R$, because then $\operatorname{rank}\left(M_{w} \lambda^{0}\right)<\operatorname{rank}\left(\lambda^{0}\right)$, in general. It ought to be expected that $\lambda^{0}$ and $f^{0}$ have to feature in the identification conditions for the low-rank regressors, because the interactive fixed effects structure and the low-rank regressors represent similar types of low-rank $N \times T$ structures.

Assumption $\operatorname{ID}(v)$ is a generalized non-collinearity assumption for the high-rank regressors, which guarantees any linear combination $\alpha \cdot X_{\text {high }}$ of the high-rank regressors is sufficiently different from the low-rank regressors and from the interactive fixed effects. A standard noncollinearity assumption can be formulated by demanding the $N \times N$ matrix $\mathbb{E}\left[\left(\alpha \cdot X_{\text {high }}\right)(\alpha\right.$. $\left.\left.X_{\text {high }}\right)^{\prime} \mid \lambda^{0}, f^{0}, w\right]$ is non-zero for all non-zero $\alpha \in \mathbb{R}^{K_{2}}$, which can be equivalently expressed as $\operatorname{rank}\left\{\mathbb{E}\left[\left(\alpha \cdot X_{\text {high }}\right)\left(\alpha \cdot X_{\text {high }}\right)^{\prime} \mid \lambda^{0}, f^{0}, w\right]\right\}>0$ for all non-zero $\alpha \in \mathbb{R}^{K_{2}}$. Assumption $\operatorname{ID}(v)$ strengthens this standard non-collinearity assumption by demanding the rank not only to be positive, but larger than $2 R+K_{1}$. This also explains the name "high-rank regressors," because their rank has to be sufficiently large to satisfy this assumption. Note also that only the number of factors $R$, but not $\lambda^{0}$ and $f^{0}$, features in Assumption $\operatorname{ID}(v)$. The sample version of this assumption is given by Assumption $4(i i)(a)$ below, which is also very closely related to Assumption A in Bai (2009).

Theorem 2.1 (Identification). Suppose the Assumptions ID are satisfied. Then, the minima of the expected objective function $\mathbb{E}\left(\left\|Y-\beta \cdot X-\lambda f^{\prime}\right\|_{F}^{2} \mid \lambda^{0}, f^{0}, w\right)$ over $(\beta, \lambda, f) \in \mathbb{R}^{K+N \times R+T \times R}$ satisfy $\beta=\beta^{0}$ and $\lambda f^{\prime}=\lambda^{0} f^{0 \prime}$. This shows that $\beta^{0}$ and $\lambda^{0} f^{0 \prime}$ are identified.

The theorem shows the true parameters are identified as minima of the expected value of $\left\|Y-\beta \cdot X-\lambda f^{\prime}\right\|_{F}^{2}=\sum_{i, t}\left(Y_{i t} \beta^{\prime}-X_{i t}-\lambda_{i}^{\prime} f_{t}\right)^{2}$, which is the sum of squared residuals. We use the same objective function, to define the estimators $\widehat{\beta}, \widehat{\lambda}$ and $\widehat{f}$ below. Without further normalization conditions, the parameters $\lambda^{0}$ and $f^{0}$ are not separately identified, because the outcome variable $Y$ is invariant under transformations $\lambda^{0} \rightarrow \lambda^{0} A^{\prime}$ and $f^{0} \rightarrow f^{0} A^{-1}$, where $A$ is a non-singular $R \times R$ matrix. However, the product $\lambda^{0} f^{0 \prime}$ is uniquely identified according to the theorem. Because our focus is on identification and estimation of $\beta^{0}$, we do not need to discuss those additional normalization conditions for $\lambda^{0}$ and $f^{0}$ in this paper. 


\section{Estimator and Consistency}

The objective function of the model is simply the sum of squared residuals, which in matrix notation can be expressed as

$$
\begin{aligned}
\mathcal{L}_{N T}(\beta, \lambda, f) & =\frac{1}{N T}\left\|Y-\beta \cdot X-\lambda f^{\prime}\right\|_{F}^{2} \\
& =\frac{1}{N T} \operatorname{Tr}\left[\left(Y-\beta \cdot X-\lambda f^{\prime}\right)^{\prime}\left(Y-\beta \cdot X-\lambda f^{\prime}\right)\right] .
\end{aligned}
$$

The estimator we consider is the LS estimator that jointly minimizes $\mathcal{L}_{N T}(\beta, \lambda, f)$ over $\beta, \lambda$ and $f$. Our main objects of interest are the regression parameters $\beta=\left(\beta_{1}, \ldots, \beta_{K}\right)^{\prime}$, whose estimator is given by

$$
\widehat{\beta}=\underset{\beta \in \mathbb{B}}{\operatorname{argmin}} L_{N T}(\beta)
$$

where $\mathbb{B} \subset \mathbb{R}^{K}$ is a compact parameter set that contains the true parameter, namely, $\beta^{0} \in \mathbb{B}$, and the objective function is the profile objective function

$$
\begin{aligned}
L_{N T}(\beta) & =\min _{\lambda, f} \mathcal{L}_{N T}(\beta, \lambda, f) \\
& =\min _{f} \frac{1}{N T} \operatorname{Tr}\left[(Y-\beta \cdot X) M_{f}(Y-\beta \cdot X)^{\prime}\right] \\
& =\frac{1}{N T} \sum_{r=R+1}^{T} \mu_{r}\left[(Y-\beta \cdot X)^{\prime}(Y-\beta \cdot X)\right] .
\end{aligned}
$$

Here, the first expression for $L_{N T}(\beta)$ is its definition as the minimum value of $\mathcal{L}_{N T}(\beta, \lambda, f)$ over $\lambda$ and $f$. We denote the minimizing incidental parameters by $\widehat{\lambda}(\beta)$ and $\widehat{f}(\beta)$, and we define the estimators $\widehat{\lambda}=\widehat{\lambda}(\widehat{\beta})$ and $\widehat{f}=\widehat{f}(\widehat{\beta})$. Those minimizing incidental parameters are not uniquely determined - for the same reason that $\lambda^{0}$ and $f^{0}$ are non uniquely identified - but the product $\widehat{\lambda}(\beta) \widehat{f}^{\prime}(\beta)$ is unique.

The second expression for $L_{N T}(\beta)$ in equation (4) is obtained by concentrating out $\lambda$ (analogously, one can concentrate out $f$ to obtain a formulation whereby only the parameter $\lambda$ remains). The optimal $f$ in the second expression is given by the $R$ eigenvectors that correspond to the $R$ largest eigenvalues of the $T \times T$ matrix $(Y-\beta \cdot X)^{\prime}(Y-\beta \cdot X)$. This insight leads to the third line that presents the profile objective function as the sum over the $T-R$ smallest eigenvalues of this $T \times T$ matrix. Lemma A.1 in the appendix shows equivalence of the three expressions for $L_{N T}(\beta)$ given above.

Multiple local minima of $L_{N T}(\beta)$ may exist, and one should use multiple starting values for the numerical optimization of $\beta$ to guarantee the true global minimum $\widehat{\beta}$ is found. 


\section{Dynamic Panel With Interactive Effects}

To show consistency of the LS estimator $\widehat{\beta}$ of the interactive fixed effect model, and also later for our first-order asymptotic theory, we consider the limit $N, T \rightarrow \infty$. In the following we present assumptions on $X_{k}, e, \lambda$, and $f$ that guarantee consistency. ${ }^{12}$

Assumption 1. (i) $\operatorname{plim}_{N, T \rightarrow \infty}\left(\lambda^{0 \prime} \lambda^{0} / N\right)>0$, (ii) $\operatorname{plim}_{N, T \rightarrow \infty}\left(f^{0 \prime} f^{0} / T\right)>0$.

Assumption 2. $\operatorname{plim}_{N, T \rightarrow \infty}\left[(N T)^{-1} \operatorname{Tr}\left(X_{k} e^{\prime}\right)\right]=0$, for all $k=1, \ldots, K$.

Assumption 3. $\operatorname{plim}_{N, T \rightarrow \infty}(\|e\| / \sqrt{N T})=0$.

Assumption 1 guarantees the matrices $f^{0}$ and $\lambda^{0}$ have full rank, that is, that $R$ distinct factors and factor loadings exist asymptotically, and that the norm of each factor and factor loading grows at a rate of $\sqrt{T}$ and $\sqrt{N}$, respectively. Assumption 2 demands the regressors are weakly exogenous. Assumption 3 restricts the spectral norm of the $N \times T$ error matrix $e$. We discuss this assumption in more detail in the next section, and we give examples of error distributions that satisfy this condition in section S.2 of the supplementary material. The final assumption needed for consistency is an assumption on the regressors $X_{k}$. We already introduced the distinction between the $K_{1}$ "low-rank regressors" $X_{l}, l=1, \ldots, K_{1}$, and the $K_{2}=K-K_{1}$ "high-rank regressors" $X_{m}, m=K_{1}+1, \ldots, K$ above.

\section{Assumption 4.}

(i) $\operatorname{plim}_{N, T \rightarrow \infty}\left[(N T)^{-1} \sum_{i=1}^{N} \sum_{t=1}^{T} X_{i t} X_{i t}^{\prime}\right]>0$.

(ii) The two types of regressors satisfy:

(a) Consider linear combinations $\alpha \cdot X_{\text {high }}=\sum_{m=K_{1}+1}^{K} \alpha_{m} X_{m}$ of the high-rank regressors $X_{m}$ for $K_{2}$-vectors $\alpha$ with $\|\alpha\|=1$, where the components of the $K_{2}$-vector $\alpha$ are denoted by $\alpha_{K_{1}+1}$ to $\alpha_{K}$. We assume a constant $b>0$ exists such that

$$
\min _{\left\{\alpha \in \mathbb{R}^{\left.K_{2},\|\alpha\|=1\right\}}\right.} \sum_{r=2 R+K_{1}+1}^{N} \mu_{r}\left[\frac{\left(\alpha \cdot X_{\text {high }}\right)\left(\alpha \cdot X_{\text {high }}\right)^{\prime}}{N T}\right] \geq b \quad \text { wpa1. }
$$

(b) For the low-rank regressors, we assume $\operatorname{rank}\left(X_{l}\right)=1, l=1, \ldots, K_{1}$; that is, they can be written as $X_{l}=w_{l} v_{l}^{\prime}$ for $N$-vectors $w_{l}$ and T-vectors $v_{l}$, and we define the $N \times K_{1}$ matrix $w=\left(w_{1}, \ldots, w_{K_{1}}\right)$ and the $T \times K_{1}$ matrix $v=\left(v_{1}, \ldots, v_{K_{1}}\right)$. We assume a constant $B>0$ exists such that $N^{-1} \lambda^{0 \prime} M_{w} \lambda^{0}>B \mathbb{I}_{R}$ and $T^{-1} f^{0 \prime} M_{v} f^{0}>B \mathbb{I}_{R}$, wpa1. 


\section{Dynamic Panel With Interactive Effects}

Assumption $4(i)$ is a standard non-collinearity condition for all the regressors. Assumption $4(i i)(a)$ is an appropriate sample analog of the identification Assumption $\operatorname{ID}(v)$. If the sum in Assumption $4(i i)(a)$ were to start from $r=1$, we would have $\sum_{r=1}^{N} \mu_{r}\left[\frac{\left(\alpha \cdot X_{\text {high }}\right)\left(\alpha \cdot X_{\text {high }}\right)^{\prime}}{N T}\right]=$ $\frac{1}{N T} \operatorname{Tr}\left[\left(\alpha \cdot X_{\text {high }}\right)\left(\alpha \cdot X_{\text {high }}\right)^{\prime}\right]$, so that the assumption would become a standard non-collinearity condition. Not including the first $2 R+K_{1}$ eigenvalues in the sum implies the $N \times N$ matrix $\left(\alpha \cdot X_{\text {high }}\right)\left(\alpha \cdot X_{\text {high }}\right)^{\prime}$ needs to have rank larger than $2 R+K_{1}$.

Assumption $4(i i)(b)$ is closely related to the identification Assumptions ID $(i i i)$ and (iv). The appearance of the factors and factor loadings in this assumption on the low-rank regressors is inevitable to guarantee consistency. For example, consider a low-rank regressor that is crosssectionally independent and proportional to the $r$ 'th unobserved factor, for example, $X_{l, i t}=f_{t r}$. The corresponding regression coefficient $\beta_{l}$ is then not identified, because the model is invariant under a shift $\beta_{l} \mapsto \beta_{l}+a, \lambda_{i r} \mapsto \lambda_{i r}-a$, for an arbitrary $a \in \mathbb{R}$. This phenomenon is well known from ordinary fixed effect models, where the coefficients of time-invariant regressors are not identified. Assumption 4(ii)(b) therefore guarantees for $X_{l}=w_{l} v_{l}^{\prime}$ that $w_{l}$ is sufficiently different from $\lambda^{0}$, and $v_{l}$ is sufficiently different from $f^{0}$.

Theorem 3.1 (Consistency). Let Assumptions 1, 2, 3, and 4 be satisfied; let the parameter set $\mathbb{B}$ be compact; and let $\beta^{0} \in \mathbb{B}$. In the limit $N, T \rightarrow \infty$, we then have

$$
\widehat{\beta} \underset{p}{\longrightarrow} \beta^{0}
$$

We assume compactness of $\mathbb{B}$ to guarantee existence of the minimizing $\widehat{\beta}$. We also use boundedness of $\mathbb{B}$ in the consistency proof, but only for those parameters $\beta_{l}, l=1 \ldots K_{1}$, that correspond to low-rank regressors, that is, if only high-rank regressors $\left(K_{1}=0\right)$ are present, the compactness assumption can be omitted, as long as existence of $\widehat{\beta}$ is guaranteed (e.g., for $\left.\mathbb{B}=\mathbb{R}^{K}\right)$.

Bai (2009) also proves consistency of the LS estimator of the interactive fixed effect model, but under somewhat different assumptions. He also employs what we call Assumptions 1 and 2, and he uses a low-level version of Assumption 3. He demands the regressors to be strictly exogenous. Regarding consistency, the main difference between our assumptions and his is the treatment of high- and low-rank regressors. He first gives a condition on the regressors (his Assumption A) that rules out low-rank regressors, and later discusses the case in which all regressors are either time-invariant or common regressors (i.e., are all low rank). By contrast, our Assumption 4 allows for a combination of high- and low-rank regressors, and for low-rank regressors that are more general than time-invariant and common regressors. 


\section{Asymptotic Distribution and Bias Correction}

Because we have already shown consistency of the LS estimator $\widehat{\beta}$, it is sufficient to study the local properties of the objective function $L_{N T}(\beta)$ around $\beta^{0}$ to derive the first-order asymptotic theory of $\widehat{\beta}$. Moon and Weidner (2015) derived a useful approximation of $L_{N T}(\beta)$ around $\beta^{0}$, and we briefly summarize the ideas and results of this approximation in the following subsection. We then apply those results to derive the asymptotic distribution of the LS estimator, including working out the asymptotic bias, which was not done previously. Afterward, we discuss bias correction and inference.

\subsection{Expansion of the Profile Objective Function}

The last expression in equation (4) for the profile objective function is convenient because it does not involve any minimization over the parameters $\lambda$ or $f$. On the other hand, this expression cannot be easily discussed by analytic means, because in general, no explicit formula exists for the eigenvalues of a matrix. The conventional method that involves a Taylor series expansion in the regression parameters $\beta$ alone seems infeasible here. In Moon and Weidner (2015), we showed how to overcome this problem by expanding the profile objective function jointly in $\beta$ and $\|e\|$. The key idea is the following decomposition:

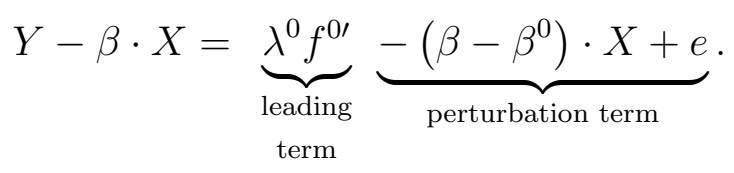

If the perturbation term is zero, the profile objective $L_{N T}(\beta)$ is also zero, because the leading term $\lambda^{0} f^{0 \prime}$ has rank $R$, so that the $T-R$ smallest eigenvalues of $f^{0} \lambda^{0 \prime} \lambda^{0} f^{0 \prime}$ all vanish. One may thus expect that small values of the perturbation term should correspond to small values of $L_{N T}(\beta)$. This idea can indeed be made mathematically precise. By using the perturbation theory of linear operators (see, e.g., Kato (1980)), one can work out an expansion of $L_{N T}(\beta)$ in the perturbation term, and one can show this expansion is convergent as long as the spectral norm of the perturbation term is sufficiently small.

The assumptions on the model made so far are in principle already sufficient to apply this expansion of the profile objective function, but to truncate the expansion at an appropriate order and to provide a bound on the remainder term that is sufficient to derive the first-order asymptotic theory of the LS estimator, we need to strengthen Assumption 3 as follows.

Assumption $3^{*}$. $\|e\|=o_{p}\left(N^{2 / 3}\right)$. 


\section{Dynamic Panel with Interactive Effects}

In the rest of the paper, we only consider asymptotics in which $N$ and $T$ grow at the same rate; that is, we could equivalently write $o_{p}\left(T^{2 / 3}\right)$ instead of $o_{p}\left(N^{2 / 3}\right)$ in Assumption $3^{*}$. In section S.2 of the supplementary material, we provide examples of error distributions that satisfy Assumption $3^{*}$. In fact, for these examples, we have $\|e\|=\mathcal{O}_{p}(\sqrt{\max (N, T)})$. A large literature studies the asymptotic behavior of the spectral norm of random matrices; see, for example, Geman (1980), Silverstein (1989), Bai, Silverstein, and Yin (1988), Yin, Bai, and Krishnaiah (1988), and Latala (2005). Loosely speaking, we expect the result $\|e\|=\mathcal{O}_{p}(\sqrt{\max (N, T)})$ to hold as long as the errors $e_{i t}$ have mean zero, uniformly bounded fourth moment, and weak time-serial and cross-sectional correlation (in some well-defined sense, see the examples).

We can now present the quadratic approximation of the profile objective function $L_{N T}(\beta)$ that we derived in Moon and Weidner (2015).

Theorem 4.1 (Expansion of Profile Objective Function). Let Assumption 1, 3*, and $4(i)$ be satisfied, and consider the limit $N, T \rightarrow \infty$ with $N / T \rightarrow \kappa^{2}, 0<\kappa<\infty$. Then, the profile objective function satisfies $L_{N T}(\beta)=L_{q, N T}(\beta)+(N T)^{-1} R_{N T}(\beta)$, where the remainder $R_{N T}(\beta)$ is such that for any sequence $\eta_{N T} \rightarrow 0$, we have

$$
\sup _{\left\{\beta:\left\|\beta-\beta^{0}\right\| \leq \eta_{N T}\right\}} \frac{\left|R_{N T}(\beta)\right|}{\left(1+\sqrt{N T}\left\|\beta-\beta^{0}\right\|\right)^{2}}=o_{p}(1),
$$

and $L_{q, N T}(\beta)$ is a second-order polynomial in $\beta$; namely,

$$
L_{q, N T}(\beta)=L_{N T}\left(\beta^{0}\right)-\frac{2}{\sqrt{N T}}\left(\beta-\beta^{0}\right)^{\prime} C_{N T}+\left(\beta-\beta^{0}\right)^{\prime} W_{N T}\left(\beta-\beta^{0}\right),
$$

with $K \times K$ matrix $W_{N T}$ defined by $W_{N T, k_{1} k_{2}}=(N T)^{-1} \operatorname{Tr}\left(M_{f^{0}} X_{k_{1}}^{\prime} M_{\lambda^{0}} X_{k_{2}}\right)$, and K-vector $C_{N T}$ with entries $C_{N T, k}=C^{(1)}\left(\lambda^{0}, f^{0}, X_{k} e\right)+C^{(2)}\left(\lambda^{0}, f^{0}, X_{k} e\right)$, where

$$
\begin{aligned}
& C^{(1)}\left(\lambda^{0}, f^{0}, X_{k}, e\right)=\frac{1}{\sqrt{N T}} \operatorname{Tr}\left(M_{f^{0}} e^{\prime} M_{\lambda^{0}} X_{k}\right), \\
& C^{(2)}\left(\lambda^{0}, f^{0}, X_{k}, e\right)=-\frac{1}{\sqrt{N T}}\left[\operatorname{Tr}\left(e M_{f^{0}} e^{\prime} M_{\lambda^{0}} X_{k} f^{0}\left(f^{0 \prime} f^{0}\right)^{-1}\left(\lambda^{0 \prime} \lambda^{0}\right)^{-1} \lambda^{0 \prime}\right)\right. \\
& +\operatorname{Tr}\left(e^{\prime} M_{\lambda^{0}} e M_{f^{0}} X_{k}^{\prime} \lambda^{0}\left(\lambda^{0 \prime} \lambda^{0}\right)^{-1}\left(f^{0 \prime} f^{0}\right)^{-1} f^{0 \prime}\right) \\
& \left.+\operatorname{Tr}\left(e^{\prime} M_{\lambda^{0}} X_{k} M_{f^{0}} e^{\prime} \lambda^{0}\left(\lambda^{0 \prime} \lambda^{0}\right)^{-1}\left(f^{0 \prime} f^{0}\right)^{-1} f^{0 \prime}\right)\right] \text {. }
\end{aligned}
$$

We refer to $W_{N T}$ and $C_{N T}$ as the approximated Hessian and the approximated score (at the true parameter $\beta^{0}$ ). The exact Hessian and the exact score (at the true parameter $\beta^{0}$ ) contain higher-order expansion terms in $e$, but the expansion up to the particular order above is sufficient to work out the first-order asymptotic theory of the LS estimator, as the following corollary shows. 


\section{Dynamic Panel With Interactive Effects}

Corollary 4.2. Let the assumptions of Theorem 3.1 and 4.1 hold; let $\beta^{0}$ be an interior point of the parameter set $\mathbb{B}$; and assume $C_{N T}=\mathcal{O}_{p}(1)$. We then have $\sqrt{N T}\left(\widehat{\beta}-\beta^{0}\right)=W_{N T}^{-1} C_{N T}+$ $o_{p}(1)=\mathcal{O}_{p}(1)$.

Combining consistency of the LS estimator and the expansion of the profile objective function

in Theorem 4.1, one obtains $\sqrt{N T} W_{N T}\left(\widehat{\beta}-\beta^{0}\right)=C_{N T}+o_{p}(1)$; see, for example, Andrews (1999). To obtain the corollary, one needs in addition that $W_{N T}$ does not become degenerate as $N, T \rightarrow \infty$; that is, the smallest eigenvalue of $W_{N T}$ should be bounded from below by a positive constant. Our assumptions already guarantee existence of such a lower bound, as is shown in the supplementary material.

Analogous to the expansions of the profile objective function $L_{N T}(\beta)$, one can also derive expansions of the projectors $M_{\widehat{\lambda}}$ and $M_{\widehat{f}}$, and those can be used to show consistency of $\widehat{\lambda}$ and $\widehat{f}$, up to normalization; see Lemma S.10.4 in the supplementary material.

\subsection{Asymptotic Distribution}

We now apply Corollary 4.2 to work out the asymptotic distribution of the LS estimator $\widehat{\beta}$. For this purpose, we need more specific assumptions on $\lambda^{0}, f^{0}, X_{k}$, and $e$.

Assumption 5. A sigma algebra $\mathcal{C}=\mathcal{C}_{N T}$ (which in the following we will refer to as the conditioning set) exists that contains the sigma algebra generated by $\lambda^{0}$ and $f^{0}$, such that

(i) $\mathbb{E}\left[e_{i t} \mid \mathcal{C} \vee \sigma\left(\left\{\left(X_{i s}, e_{i, s-1}\right), s \leq t\right\}\right)\right]=0$, for all $i, t .{ }^{13}$

(ii) $e_{i t}$ is independent over $t$, conditional on $\mathcal{C}$, for all $i$.

(iii) $\left\{\left(X_{i t}, e_{i t}\right), t=1, \ldots, T\right\}$ is independent across $i$, conditional on $\mathcal{C}$.

(iv) $\frac{1}{N T} \sum_{i=1}^{N} \sum_{t, s=1}^{T}\left|\operatorname{Cov}\left(X_{k, i t}, X_{\ell, i s} \mid \mathcal{C}\right)\right|=\mathcal{O}_{p}(1)$, for all $k, \ell=1, \ldots, K$.

(v) $\frac{1}{N T^{2}} \sum_{i=1}^{N} \sum_{t, s, u, v=1}^{T}\left|\operatorname{Cov}\left(e_{i t} \widetilde{X}_{k, i s}, e_{i u} \tilde{X}_{\ell, i v} \mid \mathcal{C}\right)\right|=\mathcal{O}_{p}(1)$, where $\tilde{X}_{k, i t}=X_{k, i t}-\mathbb{E}\left[X_{k, i t} \mid \mathcal{C}\right]$, for all $k, \ell=1, \ldots, K$.

(vi) An $\epsilon>0$ exists such that $\mathbb{E}\left(e_{i t}^{8} \mid \mathcal{C}\right)$ and $\mathbb{E}\left(\left\|X_{i t}\right\|^{8+\epsilon} \mid \mathcal{C}\right)$ and $\mathbb{E}\left\|\lambda_{i}^{0}\right\|^{4}$ and $\mathbb{E}\left\|f_{t}^{0}\right\|^{4+\epsilon}$ are bounded by a non-random constant, uniformly over $i, t$ and $N, T$.

(vii) $\beta^{0}$ is an interior point of the compact parameter set $\mathbb{B}$. 


\section{Dynamic Panel With Interactive Effects}

\section{Remarks on Assumption 5}

(1) Part $(i)$ of Assumption 5 imposes that $e_{i t}$ is a martingale difference sequence over time for a particular filtration. Conditioning on $\mathcal{C}$, the time series of $e_{i t}$ is independent over time (part $(i i)$ of the assumption) and the error term $e_{i t}$ and regressors $X_{i t}$ are crosssectionally independent (part ( $i i i)$ of the assumption), but unconditional correlation is allowed. Part $(i v)$ imposes weak time-serial correlation of $X_{i t}$. Part $(v)$ demands weak time-serial correlation of $\widetilde{X}_{k, i t}=X_{k, i t}-\mathbb{E}\left[X_{k, i t} \mid \mathcal{C}\right]$ and $e_{i t}$. Finally, parts (vi) and (vii) require bounded higher moments of the error term, regressors, factors and factor loadings, and a compact parameter set with an interior true parameter.

(2) Assumption $5(i)$ implies $\mathbb{E}\left(X_{k, i t} e_{i t} \mid \mathcal{C}\right)=0$ and $\mathbb{E}\left(X_{k, i t} e_{i t} X_{\ell, i s} e_{i s} \mid \mathcal{C}\right)=0$ for $t \neq s$. Thus, the assumption guarantees $X_{i t} e_{i t}$ is mean zero and uncorrelated over $t$, and independent across $i$, conditional on $\mathcal{C}$. Notice the conditional mean independence restriction in Assumption 5(i) is weaker than Assumption D of Bai (2009), besides sequential exogeneity. Bai imposes independence between $e_{i t}$ and $\left(\left\{X_{j s}, \lambda_{j}, f_{s}\right\}_{j, s}\right)$.

(3) Assumption 5 is sufficient for Assumption 2. To see this, notice $\operatorname{Tr}\left(X_{k} e^{\prime}\right)=\sum_{i, t} X_{k, i t} e_{i t}$, and also that the sequential exogeneity and the cross-sectional independence assumption imply $\mathbb{E}\left[\left((N T)^{-1} \sum_{i, t} X_{k, i t} e_{i t}\right)^{2} \mid \mathcal{C}\right]=(N T)^{-2} \sum_{i, t} \mathbb{E}\left[\left(X_{k, i t} e_{i t}\right)^{2} \mid \mathcal{C}\right]$. Then, together with the assumption of bounded moments, we have $(N T)^{-1} \sum_{i, t} X_{k, i t} e_{i t}=o_{p}(1)$.

(4) Assumption 5 is also sufficient for Assumption 3* (and thus for Assumption 3), because $e_{i t}$ is assumed independent over $t$ and across $i$ and has a bounded fourth moment, conditional on $\mathcal{C}$, which by using results in Latala (2005), implies the spectral norm satisfies $\|e\|=$ $\sqrt{\max (N, T)}$ as $N$ and $T$ become large; see the supplementary material.

(5) Examples of regressor processes, which satisfy Assumptions 5(iv) and (v), are discussed in the following. These examples also illuminate the role of the conditioning sigma field $\mathcal{C}$.

\section{Examples of DGPs for $X_{i t}$}

Here we provide examples of the DGPs of the regressors $X_{i t}$ that satisfy the conditions in Assumption 5. Proofs for these examples are provided in the supplementary material.

Example 1. The first example is a simple AR(1) interactive fixed effect regression:

$$
Y_{i t}=\beta^{0} Y_{i, t-1}+\lambda_{i}^{0 \prime} f_{t}^{0}+e_{i t},
$$




\section{Dynamic Panel With Interactive Effects}

where $e_{i t}$ is mean zero, independent across $i$ and $t$, and independent of $\lambda^{0}$ and $f^{0}$. Assume $\left|\beta^{0}\right|<1$ and that $e_{i t}, \lambda_{i}^{0}$, and $f_{t}^{0}$ all possess uniformly bounded moments of order $8+\epsilon$. In this case, the regressor is $X_{i t}=Y_{i t-1}=\lambda_{i}^{0 \prime} F_{t}^{0}+U_{i t}$, where $F_{t}^{0}=\sum_{s=0}^{\infty}\left(\beta^{0}\right)^{s} f_{t-1-s}^{0}$ and $U_{i t}=\sum_{s=0}^{\infty}\left(\beta^{0}\right)^{s} e_{i, t-1-s}$. For the conditioning sigma field $\mathcal{C}$ in Assumption 5, we choose $\mathcal{C}=\sigma\left(\left\{\lambda_{i}^{0}: 1 \leq i \leq N\right\},\left\{f_{t}^{0}: 1 \leq t \leq T\right\}\right)$. Conditional on $\mathcal{C}$, the only variation in $X_{i t}$ stems from $U_{i t}$, which is independent across $i$ and weakly correlated over $t$, so that Assumption 5(iv) holds. Furthermore, we have $\mathbb{E}\left(X_{i t} \mid \mathcal{C}\right)=\lambda_{i}^{0 \prime} F_{t}^{0}$ and $\widetilde{X}_{i t}=U_{i t}$, which allows us to verify Assumption $5(v)$.

This example can be generalized to a $\mathrm{VAR}(1)$ model as follows:

$$
\left(\begin{array}{c}
Y_{i t} \\
Z_{i t}
\end{array}\right)=\mathcal{B} \underbrace{\left(\begin{array}{c}
Y_{i, t-1} \\
Z_{i, t-1}
\end{array}\right)}_{=X_{i t}}+\left(\begin{array}{c}
\lambda_{i}^{0 \prime} f_{t}^{0} \\
d_{i t}
\end{array}\right)+\underbrace{\left(\begin{array}{c}
e_{i t} \\
u_{i t}
\end{array}\right)}_{=E_{i t}},
$$

where $Z_{i t}$ is an $m \times 1$ vector of additional variables and $\mathcal{B}$ is an $(m+1) \times(m+1)$ matrix of VAR parameters whose eigenvalues lie within the unit circle. The $m \times 1$ vector $d_{i t}$ and the factors $f_{t}^{0}$ and factor loadings $\lambda_{i}^{0}$ are assumed to be independent of the $(m+1) \times 1$ vector of innovations $E_{i t}$. Suppose our interest is to estimate the first row in equation (5), which corresponds exactly to our interactive fixed effects model with regressors $Y_{i, t-1}$ and $Z_{i, t-1}$. Choosing $\mathcal{C}$ to be the sigma field generated by all $f_{t}^{0}, \lambda_{i}^{0}, d_{i t}$, we obtain $\widetilde{X}_{i t}=\sum_{s=0}^{\infty} \mathcal{B}^{s} E_{i, t-1-s}$. Analogous to the AR(1) case, we then find Assumption 5(iv) and $(v)$ are satisfied in this example if the innovations $E_{i t}$ are independent across $i$ and over $t$, and have appropriate bounded moments.

Example 2. Consider a scalar $X_{i t}$ for simplicity, and let $X_{i t}=g\left(v_{i t}, \delta_{i}, h_{t}\right)$. We assume (i) $\left\{\left(e_{i t}, v_{i t}\right)_{i=1, \ldots, N ; t=1, \ldots, T}\right\} \perp\left\{\left(\lambda_{i}^{0}, \delta_{i}\right)_{i=1, \ldots, N},\left(f_{t}^{0}, h_{t}\right)_{t=1, \ldots, T}\right\}$, (ii) $\left(e_{i t}, v_{i t}, \delta_{i}\right)$ are independent across $i$ for all $t$, and (iii) $v_{i s} \perp e_{i t}$ for $s \leq t$ and all $i$. Furthermore, assume $\sup _{i t} \mathbb{E}\left|X_{i t}\right|^{8+\epsilon}<\infty$ for some positive $\epsilon$. For the conditioning sigma field $\mathcal{C}$ in Assumption 5 , we choose $\mathcal{C}=$ $\sigma\left(\left\{\lambda_{i}^{0}: 1 \leq i \leq N\right\}, \quad\left\{\delta_{i}: 1 \leq i \leq N\right\}, \quad\left\{f_{t}^{0}:-\infty \leq t \leq \infty\right\}, \quad\left\{h_{t}:-\infty \leq t \leq \infty\right\}\right)$. Furthermore, as in Hahn and Kuersteiner (2011), let $\mathcal{F}_{\tau}^{t}(i)=\mathcal{C} \vee \sigma\left(\left\{\left(e_{i s}, v_{i s}\right): \tau \leq s \leq t\right\}\right)$, and define the conditional $\alpha$-mixing coefficient on $\mathcal{C}$ :

$$
\alpha_{m}(i)=\sup _{A \in \mathcal{F}_{-\infty}^{t}(i), B \in \mathcal{F}_{t+m}^{\infty}(i)}[\mathbb{P}(A \cap B)-\mathbb{P}(A) \mathbb{P}(B) \mid \mathcal{C}]
$$

Let $\alpha_{m}=\sup _{i} \alpha_{m}(i)$, and assume $\alpha_{m}=O\left(m^{-\zeta}\right)$, where $\zeta>\frac{12 p}{4 p-1}$ for $p>4$. Then, Assumptions $5(i v)$ and $(v)$ are satisfied.

In this example, the shocks $h_{t}$ (which may contain the factors $f_{t}^{0}$ ), $\delta_{i}$ (which may contain the factor loadings $\lambda_{i}^{0}$ ), and $v_{i t}$ (which may contain past values of $e_{i t}$ ) can enter in a general non-linear way into the regressor $X_{i t}$. 


\section{Dynamic Panel With Interactive Effects}

The following assumption guarantees the limiting variance and the asymptotic bias converge to constant values.

Assumption 6. Let $\mathcal{X}_{k}=M_{\lambda^{0}} X_{k} M_{f^{0}}$, which is an $N \times T$ matrix with entries $\mathcal{X}_{k, i t}$. For each $i$ and $t$, define the $K$-vector $\mathcal{X}_{i t}=\left(\mathcal{X}_{1, i t}, \ldots, \mathcal{X}_{K, i t}\right)^{\prime}$. We assume existence of the following probability limits for all $k=1, \ldots, K$ :

$$
\begin{aligned}
W & =\operatorname{plim}_{N, T \rightarrow \infty} \frac{1}{N T} \sum_{i=1}^{N} \sum_{t=1}^{T} \mathcal{X}_{i t} \mathcal{X}_{i t}^{\prime} \\
\Omega & =\operatorname{plim}_{N, T \rightarrow \infty} \frac{1}{N T} \sum_{i=1}^{N} \sum_{t=1}^{T} e_{i t}^{2} \mathcal{X}_{i t} \mathcal{X}_{i t}^{\prime} \\
B_{1, k} & =\operatorname{plim}_{N, T \rightarrow \infty} \frac{1}{N} \operatorname{Tr}\left[P_{f^{0}} \mathbb{E}\left(e^{\prime} X_{k} \mid \mathcal{C}\right)\right] \\
B_{2, k} & =\operatorname{plim}_{N, T \rightarrow \infty} \frac{1}{T} \operatorname{Tr}\left[\mathbb{E}\left(e e^{\prime} \mid \mathcal{C}\right) M_{\lambda^{0}} X_{k} f^{0}\left(f^{0 \prime} f^{0}\right)^{-1}\left(\lambda^{0 \prime} \lambda^{0}\right)^{-1} \lambda^{0 \prime}\right] \\
B_{3, k} & =\operatorname{plim}_{N, T \rightarrow \infty} \frac{1}{N} \operatorname{Tr}\left[\mathbb{E}\left(e^{\prime} e \mid \mathcal{C}\right) M_{f^{0}} X_{k}^{\prime} \lambda^{0}\left(\lambda^{0 \prime} \lambda^{0}\right)^{-1}\left(f^{0 \prime} f^{0}\right)^{-1} f^{0 \prime}\right]
\end{aligned}
$$

where $\mathcal{C}$ is the same conditioning set that appears in Assumption 5.

Here, $W$ and $\Omega$ are $K \times K$ matrices, and we define the $K$-vectors $B_{1}, B_{2}$, and $B_{3}$ with components $B_{1, k}, B_{2, k}$ and $B_{3, k}, k=1, \ldots, K$.

Theorem 4.3 (Asymptotic Distribution). Let Assumptions 1, 4, 5, and 6 be satisfied, ${ }^{14}$ and consider the limit $N, T \rightarrow \infty$ with $N / T \rightarrow \kappa^{2}$, where $0<\kappa<\infty$. Then we have

$$
\sqrt{N T}\left(\widehat{\beta}-\beta^{0}\right) \underset{d}{\rightarrow} \mathcal{N}\left(W^{-1} B, W^{-1} \Omega W^{-1}\right)
$$

where $B=-\kappa B_{1}-\kappa^{-1} B_{2}-\kappa B_{3}$.

From Corollary 4.2, we already know the limiting distribution of $\widehat{\beta}$ is given by the limiting distribution of $W_{N T}^{-1} C_{N T}$. Note $W_{N T}=\frac{1}{N T} \sum_{i=1}^{N} \sum_{t=1}^{T} \mathcal{X}_{i t} \mathcal{X}_{i t}^{\prime}$; that is, $W$ is simply defined as the probability limit of $W_{N T}$. Assumption 4 guarantees $W$ is positive definite, as shown in the supplementary material.

Thus, the main task in proving Theorem 4.3 is to show the approximated score at the true parameter satisfies $C_{N T} \rightarrow_{d} \mathcal{N}(B, \Omega)$. We find the asymptotic variance $\Omega$ and the asymptotic bias $B_{1}$ originate from the $C^{(1)}$ term, whereas the two further bias terms $B_{2}$ and $B_{3}$ originate from the $C^{(2)}$ term of $C_{N T}$.

The bias $B_{1}$ is due to correlation of the errors $e_{i t}$ and the regressors $X_{k, i \tau}$ in the time direction (for $\tau>t$ ). This bias term generalizes the Nickell (1981) bias that occurs in dynamic models 


\section{Dynamic Panel With Interactive Effects}

with standard fixed effects, and it is not present in Bai (2009), where only strictly exogenous regressors are considered.

The other two bias terms $B_{2}$ and $B_{3}$ are already described in Bai (2009). If $e_{i t}$ is homoscedastic, that is, if $\mathbb{E}\left(e_{i t} \mid \mathcal{C}\right)=\sigma^{2}$, then $\mathbb{E}\left(e e^{\prime} \mid \mathcal{C}\right)=\sigma^{2} \mathbb{I}_{N}$ and $\mathbb{E}\left(e^{\prime} e \mid \mathcal{C}\right)=\sigma^{2} \mathbb{I}_{T}$, so that $B_{2}=0$ and $B_{3}=0$ (because the trace is cyclical and $f^{0 \prime} M_{f^{0}}=0$ and $\lambda^{0 \prime} M_{\lambda^{0}}=0$ ). Thus, $B_{2}$ is only non-zero if $e_{i t}$ is heteroscedastic across $i$, and $B_{3}$ is only non-zero if $e_{i t}$ is heteroscedastic over $t$. Correlation in $e_{i t}$ across $i$ or over $t$ would also generate non-zero bias terms of exactly the form $B_{2}$ and $B_{3}$, but is ruled out by our assumptions.

\subsection{Bias Correction}

Estimators for $W, \Omega, B_{1}, B_{2}$, and $B_{3}$ are obtained by forming suitable sample analogs and replacing the unobserved $\lambda^{0}, f^{0}$, and $e$ by the estimates $\widehat{\lambda}, \widehat{f}$, and the residuals $\widehat{e}$.

Definition 1. Let $\widehat{\mathcal{X}}_{k}=M_{\widehat{\lambda}} X_{k} M_{\widehat{f}}$. For each $i$ and $t$, define the $K$-vector $\widehat{\mathcal{X}}_{i t}=\left(\widehat{\mathcal{X}}_{1, i t}, \ldots, \widehat{\mathcal{X}}_{K, i t}\right)^{\prime}$. Let $\Gamma: \mathbb{R} \rightarrow \mathbb{R}$ be the truncation kernel defined by $\Gamma(x)=1$ for $|x| \leq 1$, and $\Gamma(x)=0$ otherwise. Let $M$ be a bandwidth parameter that depends on $N$ and $T$. We define the $K \times K$ matrices $\widehat{W}$ and $\widehat{\Omega}$, and the $K$-vectors $\widehat{B}_{1}, \widehat{B}_{2}$, and $\widehat{B}_{3}$ as follows:

$$
\begin{aligned}
\widehat{W} & =\frac{1}{N T} \sum_{i=1}^{N} \sum_{t=1}^{T} \widehat{\mathcal{X}}_{i t} \widehat{\mathcal{X}}_{i t}^{\prime}, \\
\widehat{\Omega} & =\frac{1}{N T} \sum_{i=1}^{N} \sum_{t=1}^{T}\left(\widehat{e}_{i t}\right)^{2} \widehat{\mathcal{X}}_{i t} \widehat{\mathcal{X}}_{i t}^{\prime}, \\
\widehat{B}_{1, k} & =\frac{1}{N} \sum_{i=1}^{N} \sum_{t=1}^{T-1} \sum_{s=t+1}^{T} \Gamma\left(\frac{s-t}{M}\right)\left[P_{\widehat{f}}\right]_{t s} \widehat{e}_{i t} X_{k, i s}, \\
\widehat{B}_{2, k} & =\frac{1}{T} \sum_{i=1}^{N} \sum_{t=1}^{T}\left(\widehat{e}_{i t}\right)^{2}\left[M_{\widehat{\lambda}} X_{k} \widehat{f}\left(\widehat{f}^{\prime} \widehat{f}\right)^{-1}\left(\widehat{\lambda}^{\prime} \widehat{\lambda}\right)^{-1} \widehat{\lambda}^{\prime}\right]_{i i}, \\
\widehat{B}_{3, k} & =\frac{1}{N} \sum_{i=1}^{N} \sum_{t=1}^{T}\left(\widehat{e}_{i t}\right)^{2}\left[M_{\widehat{f}} X_{k}^{\prime} \widehat{\lambda}\left(\widehat{\lambda}^{\prime} \widehat{\lambda}\right)^{-1}\left(\widehat{f}^{\prime} \widehat{f}\right)^{-1} \widehat{f}^{\prime}\right]_{t t},
\end{aligned}
$$

where $\widehat{e}=Y-\widehat{\beta} \cdot X-\widehat{\lambda} \widehat{f}^{\prime}$, and $\widehat{e}_{i t}$ denotes the elements of $\widehat{e},[A]_{t s}$ denotes the $(t, s)$ th element of the matrix $A$.

Notice the estimators $\widehat{\Omega}, \widehat{B}_{2}$, and $\widehat{B}_{3}$ are similar to White's standard error estimator under heteroskedasticity, and the estimator $\widehat{B}_{1}$ is similar to the HAC estimator with a kernel. To show 


\section{Dynamic Panel With Interactive Effects}

consistency of these estimators, we impose some additional assumptions.

\section{Assumption 7.}

(i) $\left\|\lambda_{i}^{0}\right\|$ and $\left\|f_{t}^{0}\right\|$ are uniformly bounded over $i$, $t$, and $N, T$.

(ii) There exist $c>0$ and $\epsilon>0$ such that for all $i, t, m, N$, and $T$, we have $\left|\frac{1}{N} \sum_{i=1}^{N} \mathbb{E}\left(e_{i t} X_{k, i t+m} \mid \mathcal{C}\right)\right| \leq c m^{-(1+\epsilon)}$.

Assumption $7(i)$ is made for convenience to simplify the consistency proof for the estimators in Definition 1. Weakening this assumption is possible by only assuming suitable bounded moments of $\left\|\lambda_{i}^{0}\right\|$ and $\left\|f_{t}^{0}\right\|$. To show consistency of $\widehat{B}_{1}$, we need to control how strongly $e_{i t}$ and $X_{k, i \tau}, t<\tau$, are allowed to be correlated, which is done by Assumption $7(i i)$. It is straightforward to verify Assumption $7(i i)$ is satisfied in the two examples of regressor processes presented after Assumption 5.

Theorem 4.4 (Consistency of Bias and Variance Estimators). Let Assumptions 1, 4, 5, 6, and 7 hold, and consider a limit $N, T \rightarrow \infty$ with $N / T \rightarrow \kappa^{2}, 0<\kappa<\infty$, such that the bandwidth $M=M_{N T}$ satisifies $M \rightarrow \infty$ and $M^{5} / T \rightarrow 0$. We then have $\widehat{W}=W+o_{p}(1)$, $\widehat{\Omega}=\Omega+o_{p}(1), \widehat{B}_{1}=B_{1}+o_{p}(1), \widehat{B}_{2}=B_{2}+o_{p}(1)$, and $\widehat{B}_{3}=B_{3}+o_{p}(1)$.

The assumption $M^{5} / T \rightarrow 0$ can be relaxed if additional higher- moment restrictions on $e_{i t}$ and $X_{k, i t}$ are imposed. Note also that for the construction of the estimators $\widehat{W}, \widehat{\Omega}$, and $\widehat{B}_{i}, i=$ $1,2,3$, knowing whether the regressors are strictly exogenous or predetermined is unnecessary; in both cases, the estimators for $W, \Omega$, and $B_{i}, i=1,2,3$, are consistent. We can now present our bias-corrected estimator and its limiting distribution.

Corollary 4.5. Under the assumptions of Theorem 4.4, the bias-corrected estimator

$$
\widehat{\beta}^{*}=\widehat{\beta}+\widehat{W}^{-1}\left(T^{-1} \widehat{B}_{1}+N^{-1} \widehat{B}_{2}+T^{-1} \widehat{B}_{3}\right)
$$

satisfies $\sqrt{N T}\left(\widehat{\beta}^{*}-\beta^{0}\right) \rightarrow_{d} \mathcal{N}\left(0, W^{-1} \Omega W^{-1}\right)$.

According to Theorem 4.4, a consistent estimator of the asymptotic variance of $\widehat{\beta}^{*}$ is given by $\widehat{W}^{-1} \widehat{\Omega} \widehat{W}^{-1}$.

An alternative to the analytical bias-correction result given by Corollary 4.5 is to use Jackknife bias correction to eliminate the asymptotic bias. For panel models with incidental parameters only in the cross-sectional dimensions, one typical finds a large $N, T$ leading incidental parameter bias of order $1 / T$ for the parameters of interest. To correct for this $1 / T$ bias, one 


\section{Dynamic Panel With Interactive Effects}

can use the delete-one Jackknife bias correction if observations are iid over $t$ (Hahn and Newey, 2004) and the split-panel Jackknife bias-correction if observations are correlated over $t$ (Dhaene and Jochmans, 2015). In our current model, we have incidental parameters in both panel dimensions $\left(\lambda_{i}^{0}\right.$ and $\left.f_{t}^{0}\right)$, resulting in leading bias terms of order $1 / T$ (bias term $B_{1}$ and $B_{3}$ ) and of order $1 / N$ (bias term $B_{2}$ ). Fernández-Val and Weidner (2013) discuss the generalizations of the split-panel Jackknife bias-correction to that case.

The corresponding bias-corrected split-panel Jackknife estimator reads $\widehat{\beta}^{J}=3 \widehat{\beta}_{N T}-\bar{\beta}_{N, T / 2}-$ $\bar{\beta}_{N / 2, T}$, where $\widehat{\beta}_{N T}=\widehat{\beta}$ is the LS estimator obtained from the full sample, $\bar{\beta}_{N, T / 2}$ is the average of the two LS estimators that leave out the first and second halves of the time periods, and $\bar{\beta}_{N / 2, T}$ is the average of the two LS estimators that leave out half of the individuals. Jackknife bias correction is convenient because only the order of the bias, and not the structure of the terms $B_{1}, B_{2}$, and $B_{3}$, needs not be known in detail. However, one requires additional stationarity assumptions over $t$ and homogeneity assumptions across $i$ to justify the Jackknife correction and to show that $\widehat{\beta}^{J}$ has the same limiting distribution as $\widehat{\beta}^{*}$ in Corollary 4.5; see Fernández-Val and Weidner (2013) for more details. They also observe through Monte Carlo simulations that the finite sample variance of the Jackknife-corrected estimator is often larger than of the analytically corrected estimator. We do not explore Jackknife bias-correction further in this paper.

\section{$5 \quad$ Testing Restrictions on $\beta^{0}$}

In this section, we discuss the three classical test statistics for testing linear restrictions on $\beta^{0}$. The null hypothesis is $H_{0}: H \beta^{0}=h$, and the alternative is $H_{a}: H \beta^{0} \neq h$, where $H$ is an $r \times K$ matrix of rank $r \leq K$, and $h$ is an $r \times 1$ vector. We restrict the presentation to testing a linear hypothesis for ease of exposition. One can generalize the discussion to the testing of non-linear hypotheses, under conventional regularity conditions. Throughout this subsection, we assume $\beta^{0}$ is an interior point of $\mathbb{B}$; that is, no local restrictions are on $\beta$ as long as the null hypothesis is not imposed. Using the expansion of $L_{N T}(\beta)$, one could also discuss testing when the true parameter is on the boundary, as shown in Andrews (2001).

The restricted estimator is defined by

$$
\widetilde{\beta}=\underset{\beta \in \widetilde{\mathbb{B}}}{\operatorname{argmin}} L_{N T}(\beta),
$$

where $\widetilde{\mathbb{B}}=\{\beta \in \mathbb{B} \mid H \beta=h\}$ is the restricted parameter set. Analogous to Theorem 4.3 for the unrestricted estimator $\widehat{\beta}$, we can use the expansion of the profile objective function to derive the limiting distribution of the restricted estimator. Under the assumptions of Theorem 4.3, we 
have

$$
\sqrt{N T}\left(\widetilde{\beta}-\beta^{0}\right) \underset{d}{\longrightarrow} \mathcal{N}\left(\mathfrak{W}^{-1} B, \mathfrak{W}^{-1} \Omega \mathfrak{W}^{-1}\right)
$$

where $\mathfrak{W}^{-1}=W^{-1}-W^{-1} H^{\prime}\left(H W^{-1} H^{\prime}\right)^{-1} H W^{-1}$. The $K \times K$ covariance matrix in the limiting distribution of $\widetilde{\beta}$ is not full rank, but satisfies $\operatorname{rank}\left(\mathfrak{W}^{-1} \Omega \mathfrak{W}^{-1}\right)=K-r$, because $H \mathfrak{W}^{-1}=0$ and thus $\operatorname{rank}\left(\mathfrak{W}^{-1}\right)=K-r$. The asymptotic distribution of $\sqrt{N T}\left(\widetilde{\beta}-\beta^{0}\right)$ is therefore $K-r$ dimensional, as it should be for the restricted estimator.

\section{Wald Test}

Using the result of Theorem 4.3, we find that under the null hypothesis, $\sqrt{N T}(H \widehat{\beta}-h)$ is asymptotically distributed as $\mathcal{N}\left(H W^{-1} B, H W^{-1} \Omega W^{-1} H^{\prime}\right)$. Thus, due to the presence of the bias $B$, the standard Wald test statistic $W D_{N T}=N T(H \widehat{\beta}-h)^{\prime}\left(H \widehat{W}^{-1} \widehat{\Omega} \widehat{W}^{-1} H^{\prime}\right)^{-1}(H \widehat{\beta}-h)$ is not asymptotically $\chi_{r}^{2}$ distributed. Using the estimator $\widehat{B}=-\sqrt{\frac{N}{T}} \widehat{B}_{1}-\sqrt{\frac{T}{N}} \widehat{B}_{2}-\sqrt{\frac{N}{T}} \widehat{B}_{3}$ for the bias, we can define the bias-corrected Wald test statistic as

$$
W D_{N T}^{*}=\left[\sqrt{N T}\left(H \widehat{\beta}^{*}-h\right)\right]^{\prime}\left(H \widehat{W}^{-1} \widehat{\Omega} \widehat{W}^{-1} H^{\prime}\right)^{-1}\left[\sqrt{N T}\left(H \widehat{\beta}^{*}-h\right)\right],
$$

where $\widehat{\beta}^{*}=\widehat{\beta}-\widehat{W}^{-1} \widehat{B}$ is the bias-corrected estimator. $W D_{N T}^{*}$ is just the standard Wald test

statistics applied to $\widehat{\beta}^{*}$. Under the null hypothesis and the Assumptions of Theorem 4.4, we find $W D_{N T}^{*} \rightarrow_{d} \chi_{r}^{2}$.

\section{Likelihood Ratio Test}

To implement the LR test, we need the relationship between the asymptotic Hessian $W$ and the asymptotic score variance $\Omega$ of the profile objective function to be of the form $\Omega=c W$, where $c>0$ is a scalar constant. This condition is satisfied in our interactive fixed effect model if $\mathbb{E}\left(e_{i t}^{2} \mid \mathcal{C}\right)=c$, that is, if the error is homoskedastic. A consistent estimator for $c$ is then given by $\widehat{c}=(N T)^{-1} \sum_{i=1}^{N} \sum_{t=1}^{T} \widehat{e}_{i t}^{2}$, where $\widehat{e}=Y-\widehat{\beta} \cdot X-\widehat{\lambda} \widehat{f}^{\prime}$. Because the likelihood function for the interactive fixed effect model is just the sum of squared residuals, we have $\widehat{c}=L_{N T}(\widehat{\beta})$. The likelihood ratio test statistic is defined by

$$
L R_{N T}=\widehat{c}^{-1} N T\left[L_{N T}(\widetilde{\beta})-L_{N T}(\widehat{\beta})\right] .
$$

Under the assumption of Theorem 4.3, we then have

$$
L R_{N T} \underset{d}{\longrightarrow} \quad c^{-1} C^{\prime} W^{-1} H^{\prime}\left(H W^{-1} H^{\prime}\right)^{-1} H W^{-1} C
$$




\section{Dynamic Panel With Interactive Effects}

where $C \sim \mathcal{N}(B, \Omega)$, i.e. $C_{N T} \rightarrow{ }_{d} C$. It is the same limiting distribution that one finds for the Wald test if $\Omega=c W$ (in fact, one can show $W D_{N T}=L R_{N T}+o_{p}(1)$ ). Therefore, we need to do a bias-correction for the LR test to achieve a $\chi^{2}$ limiting distribution. We define

$$
L R_{N T}^{*}=\widehat{c}^{-1} N T\left[\min _{\{\beta \in \mathbb{B} \mid H \beta=h\}} L_{N T}\left(\beta+(N T)^{-1 / 2} \widehat{W}^{-1} \widehat{B}\right)-\min _{\beta \in \mathbb{B}} L_{N T}\left(\beta+(N T)^{-1 / 2} \widehat{W}^{-1} \widehat{B}\right)\right],
$$

where $\widehat{B}$ and $\widehat{W}$ do not depend on the parameter $\beta$ in the minimization problem. ${ }^{15}$ Asymp-

totically, we have $\min _{\beta \in \mathbb{B}} L_{N T}\left(\beta+(N T)^{-1 / 2} \widehat{W}^{-1} \widehat{B}\right)=L_{N T}(\widehat{\beta})$, because $\beta \in \mathbb{B}$ does not impose local constraints; in other words, close to $\beta^{0}$, whether one minimizes over $\beta$ or over $\beta+(N T)^{-1 / 2} \widehat{W}^{-1} \widehat{B}$ does not matter for the value of the minimum. The correction to the LR test therefore originates from the first term in $L R_{N T}^{*}$. For the minimization over the restricted parameter set, whether the argument of $L_{N T}$ is $\beta$ or $\beta+(N T)^{-1 / 2} \widehat{W}^{-1} \widehat{B}$ matters, because generically, we have $H W^{-1} B \neq 0$ (otherwise, no correction would be necessary for the LR statistics). One can show that

$$
L R_{N T}^{*} \underset{d}{\longrightarrow} c^{-1}(C-B)^{\prime} W^{-1} H^{\prime}\left(H W^{-1} H^{\prime}\right)^{-1} H W^{-1}(C-B) ;
$$

that is, we obtain the same formula as for $L R_{N T}$, but the bias-corrected term $C-B$ replaces the limit of the score $C$. Under the Assumptions of Theorem 4.4, if $H_{0}$ is satisfied, and for homoscedastic errors $e_{i t}$, we have $L R_{N T}^{*} \rightarrow_{d} \chi_{r}^{2}$. In fact, one can show $L R_{N T}^{*}=W D_{N T}^{*}+o_{p}(1)$.

\section{Lagrange Multiplier Test}

Let $\widetilde{\nabla} \mathcal{L}_{N T}$ be the gradient of the LS objective function (2) with respect to $\beta$, evaluated at the restricted parameter estimates; that is,

$$
\begin{aligned}
\widetilde{\nabla} \mathcal{L}_{N T}=\nabla \mathcal{L}_{N T}(\widetilde{\beta}, \tilde{\lambda}, \widetilde{f}) & =\left(\left.\frac{\partial \mathcal{L}_{N T}(\beta, \widetilde{\lambda}, \widetilde{f})}{\partial \beta_{1}}\right|_{\beta=\widetilde{\beta}}, \ldots,\left.\frac{\partial \mathcal{L}_{N T}(\beta, \widetilde{\lambda}, \widetilde{f})}{\partial \beta_{K}}\right|_{\beta=\tilde{\beta}}\right)^{\prime} \\
& =-\frac{2}{N T}\left(\operatorname{Tr}\left(X_{1}^{\prime} \widetilde{e}\right), \ldots, \operatorname{Tr}\left(X_{K}^{\prime} \widetilde{e}\right)\right)^{\prime}
\end{aligned}
$$

where $\widetilde{\lambda}=\widehat{\lambda}(\widetilde{\beta}), \widetilde{f}=\widehat{f}(\widetilde{\beta})$, and $\widetilde{e}=Y-\widetilde{\beta} \cdot X-\widetilde{\lambda} \tilde{f}^{\prime}$. Under the assumptions of Theorem 4.3, and if the null hypothesis $H_{0}: H \beta^{0}=h$ is satisfied, one finds that ${ }^{16}$

$$
\sqrt{N T} \widetilde{\nabla} \mathcal{L}_{N T}=\sqrt{N T} \nabla L_{N T}(\widetilde{\beta})+o_{p}(1)
$$

Due to this equation, one can base the Lagrange multiplier test on the gradient of $\mathcal{L}_{N T}(\widetilde{\beta}, \widetilde{\lambda}, \widetilde{f})$, or on the gradient of the profile quasi-likelihood function $L_{N T}(\widetilde{\beta})$, and obtain the same limiting distribution. 


\section{Dynamic Panel With Interactive Effects}

Using the bound on the remainder $R_{N T}(\beta)$ given in Theorem 4.1, one cannot infer any properties of the score function, that is, of the gradient $\nabla L_{N T}(\beta)$, because nothing is said about $\nabla R_{N T}(\beta)$. The following theorem gives a bound on $\nabla R_{N T}(\beta)$ that is sufficient to derive the limiting distribution of the Lagrange multiplier.

Theorem 5.1. Under the assumptions of Theorem 4.1, and with $W_{N T}$ and $C_{N T}$ as defined there, the score function satisfies

$$
\nabla L_{N T}(\beta)=2 W_{N T}\left(\beta-\beta^{0}\right)-\frac{2}{\sqrt{N T}} C_{N T}+\frac{1}{N T} \nabla R_{N T}(\beta),
$$

where the remainder $\nabla R_{N T}(\beta)$ satisfies for any sequence $\eta_{N T} \rightarrow 0$ :

$$
\sup _{\left\{\beta:\left\|\beta-\beta^{0}\right\| \leq \eta_{N T}\right\}} \frac{\left\|\nabla R_{N T}(\beta)\right\|}{\sqrt{N T}\left(1+\sqrt{N T}\left\|\beta-\beta^{0}\right\|\right)}=o_{p}(1) .
$$

From this theorem, and the fact that $\widetilde{\beta}$ is $\sqrt{N T}$-consistent under $H_{0}$, we obtain

$$
\begin{aligned}
\sqrt{N T} \widetilde{\nabla} \mathcal{L}_{N T} & =\sqrt{N T} \nabla L_{q, N T}(\widetilde{\beta})+o_{p}(1) \\
& =2 \sqrt{N T} W_{N T}\left(\widetilde{\beta}-\beta^{0}\right)-2 C_{N T}+o_{p}(1) .
\end{aligned}
$$

Remember $\widetilde{\beta}$ is the restricted estimator defined in equation (6). Using this result and the known limiting distribution of $\widetilde{\beta}$, we now find

$$
\sqrt{N T} \widetilde{\nabla} \mathcal{L}_{N T} \underset{d}{\longrightarrow}-2 H^{\prime}\left(H W^{-1} H^{\prime}\right)^{-1} H W^{-1} C
$$

Note also that $\sqrt{N T} H W^{-1} \nabla L_{N T}(\widetilde{\beta}) \rightarrow_{d}-2 H W^{-1} C$. We define $\widetilde{B}, \widetilde{W}$, and $\widetilde{\Omega}$, analogous to $\widehat{B}, \widehat{W}$, and $\widehat{\Omega}$, but with unrestricted parameter estimates replaced by restricted parameter estimates. The LM test statistic is then given by

$$
L M_{N T}=\frac{N T}{4}\left(\widetilde{\nabla} \mathcal{L}_{N T}\right)^{\prime} \widetilde{W}^{-1} H^{\prime}\left(H \widetilde{W}^{-1} \widetilde{\Omega} \widetilde{W}^{-1} H^{\prime}\right)^{-1} H \widetilde{W^{-1}} \widetilde{\nabla} \mathcal{L}_{N T}
$$

One can show the LM test is asymptotically equivalent to the Wald test: $L M_{N T}=W D_{N T}+$ $o_{p}(1)$; that is, again, bias-correction is necessary. We define the bias-corrected LM test statistic as

$$
L M_{N T}^{*}=\frac{1}{4}\left(\sqrt{N T} \widetilde{\nabla} \mathcal{L}_{N T}+2 \widetilde{B}\right)^{\prime} \widetilde{W}^{-1} H^{\prime}\left(H \widetilde{W}^{-1} \widetilde{\Omega} \widetilde{W}^{-1} H^{\prime}\right)^{-1} H \widetilde{W}^{-1}\left(\sqrt{N T} \widetilde{\nabla} \mathcal{L}_{N T}+2 \widetilde{B}\right) .
$$

The following theorem summarizes the main results of the present subsection. 
Theorem 5.2 (Chi-Square Limit of Bias-Corrected Test Statistics). Let the assumptions of Theorem 4.4 and the null hypothesis $H_{0}: H \beta^{0}=h$ be satisfied. For the bias-corrected Wald and LM test statistics introduced in equation (7) and (11), we then have

$$
W D_{N T}^{*} \underset{d}{\longrightarrow} \chi_{r}^{2}, \quad L M_{N T}^{*} \underset{d}{\longrightarrow} \chi_{r}^{2}
$$

If, in addition, we assume $\mathbb{E}\left(e_{i t}^{2} \mid \mathcal{C}\right)=c$, that is, the idiosyncratic errors are homoscedastic, and we use $\widehat{c}=L_{N T}(\widehat{\beta})$ as an estimator for $c$, the LR test statistic defined in equation (8) satisfies

$$
L R_{N T}^{*} \underset{d}{\longrightarrow} \chi_{r}^{2}
$$

\section{Extension to Endogenous Regressors}

In this section, we briefly discuss how to estimate the regression coefficient $\beta^{0}$ of Model (1) when some of the regressors in $X_{i t}$ are endogenous with respect to the regression error $e_{i t}$. The question is how instrumental variables can be used to estimate the regression coefficients of the endogenous regressor in the presence of the interactive fixed effects $\lambda_{i}^{0 \prime} f_{t}^{0}$.

The existing literature has already investigated similar questions under various setups. Harding and Lamarche $(2009 ; 2011)$ investigate the problem of estimating an endogenous panel (quantile) regression with interactive fixed effects, and show how to use IVs in the CCE estimation framework. Moon, Shum, and Weidner (2012) (hereafter MSW) estimate a random coefficient multinomial demand model (as in Berry, Levinsohn, and Pakes (1995)) when the unobserved product-market characteristics have interactive fixed effects. The IVs are required to identify the parameters of the random coefficient distribution and to control for price endogeneity. They suggested a multi-step "least squares-minimum distance" (LS-MD) estimator. ${ }^{17}$ The LS-MD approach is also applicable to linear panel regression models with endogenous regressors and interactive fixed effects, as demonstrated in Lee, Moon, and Weidner (2012) for the case of a dynamic linear panel regression model with interactive fixed effects and measurement error.

We now discuss how to implement the LS-MD estimation in our setup. Let $X_{i t}^{\text {end }}$ be the vectors of endogenous regressors, and let $X_{i t}^{\text {exo }}$ be the vector of exogenous regressors, with respect to $e_{i t}$, such that $X_{i t}=\left(X_{i t}^{\text {end }}, X_{i t}^{\text {exo' }}\right)^{\prime}$. The model then reads

$$
Y_{i t}=\beta_{\mathrm{end}}^{0 \prime} X_{i t}^{\mathrm{end}}+\beta_{\mathrm{exo}}^{0 \prime} X_{i t}^{\mathrm{exo}}+\lambda_{i}^{0 \prime} f_{t}^{0}+e_{i t},
$$

where $X_{i t}^{\text {exo }}$ denotes the exogenous and $X_{i t}^{\text {end }}$ denotes the endogenous regressors (wrt to $e_{i t}$ ). Suppose $Z_{i t}$ is an additional $L$-vector of exogenous instrumental variables (IVs), but $Z_{i t}$ may be 


\section{Dynamic Panel With Interactive Effects}

correlated with $\lambda_{i}^{0}$ and $f_{t}^{0}$. The LS-MD estimator of $\beta^{0}=\left(\beta_{\text {end }}^{0 \prime}, \beta_{\text {exo }}^{0 \prime}\right)^{\prime}$ can then be calculated by the following three steps:

(1) For given $\beta_{\text {end }}$, we run the least squares regression of $Y_{i t}-\beta_{\text {end }}^{\prime} X_{i t}^{\text {end }}$ on the included exogeneous regressors $X_{i t}^{\text {exo }}$, the interactive fixed effects $\lambda_{i}^{\prime} f_{t}$, and the IVs $Z_{i t}$ :

$$
\begin{aligned}
& \left(\widetilde{\beta}_{\text {exo }}\left(\beta_{\text {end }}\right), \widetilde{\gamma}\left(\beta_{\text {end }}\right), \widetilde{\lambda}\left(\beta_{\text {end }}\right), \widetilde{f}\left(\beta_{\text {end }}\right)\right) \\
& \quad=\underset{\left\{\beta_{\text {exo }}, \gamma, \lambda, f\right\}}{\operatorname{argmin}} \sum_{i=1}^{N} \sum_{t=1}^{T}\left(Y_{i t}-\beta_{\text {end }}^{\prime} X_{i t}^{\text {end }}-\beta_{\text {exo }}^{\prime} X_{i t}^{\text {exo }}-\gamma^{\prime} Z_{i t}-\lambda_{i}^{\prime} f_{t}\right)^{2} .
\end{aligned}
$$

(2) We estimate $\beta_{\text {end }}$ by finding $\widetilde{\gamma}\left(\beta_{\text {end }}\right)$, obtained by step (1), that is closest to zero. To do so, we choose a symmetric positive definite $L \times L$ weight matrix $W_{N T}^{\gamma}$ and compute

$$
\widehat{\beta}_{\text {end }}=\underset{\beta_{\text {end }}}{\operatorname{argmin}} \widetilde{\gamma}\left(\beta_{\text {end }}\right)^{\prime} W_{N T}^{\gamma} \widetilde{\gamma}\left(\beta_{\text {end }}\right)
$$

(3) We estimate $\beta_{\text {exo }}$ (and $\left.\lambda, f\right)$ by running the least squares regression of $Y_{i t}-\widehat{\beta}_{\text {end }}^{\prime} X_{i t}^{\text {end }}$ on the included exogeneous regressors $X_{i t}^{\text {exo }}$ and the interactive fixed effects $\lambda_{i}^{\prime} f_{t}$ :

$$
\left(\widehat{\beta}_{\text {exo }}, \widehat{\lambda}, \widehat{f}\right)=\underset{\left\{\beta_{\text {exo }}, \gamma, \lambda, f\right\}}{\operatorname{argmin}} \sum_{i=1}^{N} \sum_{t=1}^{T}\left(Y_{i t}-\widehat{\beta}_{\text {end }}^{\prime} X_{i t}^{\text {end }}-\beta_{\text {exo }}^{\prime} X_{i t}^{\text {exo }}-\lambda_{i}^{\prime} f_{t}\right)^{2}
$$

The idea behind this estimation procedure is that valid instruments are excluded from the model for $Y_{i t}$, so that their first-step regression coefficients $\widetilde{\gamma}\left(\beta_{\text {end }}\right)$ should be close to zero if $\beta_{\text {end }}$ is close to its true value $\beta_{\text {end }}^{0}$. Thus, as long as $X_{i t}^{\text {exo }}$ and $Z_{i t}$ jointly satisfy the assumptions of the current paper, we obtain $\widetilde{\gamma}\left(\beta_{\text {end }}^{0}\right)=o_{p}(1)$ for the first-step LS estimator, and we also obtain the asymptotic distribution of $\widetilde{\gamma}\left(\beta_{\text {end }}^{0}\right)$ from the results derived in section 4 .

However, to justify the second-step minimization formally, one needs to study the properties of $\widetilde{\gamma}\left(\beta_{\text {end }}\right)$ also for $\beta_{\text {end }} \neq \beta_{\text {end }}^{0}$. To do so, we refer to MSW. Our $\beta_{\text {end }}, \beta_{\text {exo }}$, and $Y_{i t}-\beta_{\text {end }}^{\prime} X_{i t}^{\text {end }}$ correspond to their $\alpha, \beta$, and $\delta_{j t}(\alpha)$, respectively. Assumptions 1 - 5 in MSW can be translated accordingly, and the results in MSW show large $N, T$ consistency and asymptotic normality of the LS-MD estimator.

The final step of the LS-MD estimation procedure is essentially a repetition of the first step, but without including $Z_{i t}$ in the set of regressors, which results in some efficiency gains for $\widehat{\beta}_{\text {exo }}$ compared to the first step. 


\section{Dynamic Panel With Interactive Effects}

\section{Monte Carlo Simulations}

We consider an $\mathrm{AR}(1)$ model with $R=1$ factors:

$$
Y_{i t}=\rho^{0} Y_{i, t-1}+\lambda_{i}^{0} f_{t}^{0}+e_{i t}
$$

We estimate the model as an interactive fixed effect model; that is, no distributional assumptions on $\lambda_{i}^{0}$ and $f_{t}^{0}$ are made in estimation. The parameter of interest is $\rho^{0}$. The estimators we consider are the OLS estimator (which completely ignores the presence of the factors), the least squares estimator with interactive fixed effects (denoted FLS in this section to differentiate from OLS) defined in equation (3), ${ }^{18}$ and its bias-corrected version (denoted BC-FLS), defined in Theorem 4.5 .

For the simulation, we draw the $e_{i t}$ independently and identically distributed from a tdistribution with five degrees of freedom, the $\lambda_{i}^{0}$ independently distributed from $\mathcal{N}(1,1)$, and we generate the factors from an $\mathrm{AR}(1)$ specification, namely, $f_{t}^{0}=\rho_{f} f_{t-1}^{0}+u_{t}$, where $u_{t} \sim$ $\operatorname{iid} \mathcal{N}\left(0,\left(1-\rho_{f}^{2}\right) \sigma_{f}^{2}\right)$, and $\sigma_{f}$ is the standard deviation of $f_{t}^{0}$. For all simulations, we generate 1,000 initial time periods for $f_{t}^{0}$ and $Y_{i t}$ that are not used for estimation. This approach guarantees the simulated data used for estimation are distributed according to the stationary distribution of the model.

This setup contains no correlation and heteroscedasticity in $e_{i t}$; that is, only the bias term $B_{1}$ of the FLS estimator is non-zero, but we ignore this information in the estimation; that is, we correct for all three bias terms $\left(B_{1}, B_{2}\right.$, and $B_{3}$, as introduced in Assumption 6$)$ in the bias-corrected FLS estimator.

Table 1 shows the simulation results for the bias, standard error, and root mean square error of the three different estimators for the case $N=100, \rho_{f}=0.5$, and $\sigma_{f}=0.5$, and different values of $\rho^{0}$ and $T$. The OLS estimator, the FLS estimator (computed with correct $R=1$ ), and the corresponding bias-corrected FLS estimator with factors (BC-FLS) were computed for 10,000 simulation runs. The table lists the mean bias, the standard deviation (std), and the square root of the mean square error (rmse) for the three estimators. As expected, the OLS estimator is biased because of the factor structure and its bias does not vanish (it actually increases) as $T$ increases. The FLS estimator is also biased, but as theory predicts its bias vanishes as $T$ increases. The bias-corrected FLS estimator performs better than the non-corrected FLS estimator, in particular, its bias vanishes faster. Because we only correct for the first-order bias of the FLS estimator, we could not expect the bias-corrected FLS estimator to be unbiased. However, as $T$ gets larger, more and more of the FLS estimator bias is corrected for; for example, for $\rho^{0}=0.3$, we find that at $T=5$, the bias correction only corrects for about half of the bias, 


\section{Dynamic Panel With Interactive Effects}

whereas at $T=80$, it already corrects for about $90 \%$ of it.

Table 2 is similar to Table 1, with the only difference being that we allow for misspecification in the number of factors $R$, namely, the true number of factors is assumed to be $R=1$ (i.e., same DGP as for Table 1), but we incorrectly use $R=2$ factors when calculating the FLS and BC-FLS estimator. By comparing Table 2 with Table 1, we find this type of misspecification of the number of factors increases the bias and the standard deviation of both the FLS and the BC-FLS estimator in finite samples. That increase, however, is comparatively small once both $N$ and $T$ are large. According to the results in Moon and Weidner (2015), we expect the limiting distribution of the correctly specified $(R=1)$ and incorrectly specified $(R=2)$ FLS estimator to be identical when $N$ and $T$ grow at the same rate. Our simulations suggest the same is true for the BC-FLS estimator. The remaining simulation all assume correctly specified $R=1$.

An import issue is the choice of bandwidth $M$ for the bias correction. Table 3 gives the fraction of the FLS estimator bias that is captured by the estimator for the bias in a model with $N=100, T=20, \rho_{f}=0.5, \sigma_{f}=0.5$ and different values for $\rho$ and $M$. The table shows the optimal bandwidth (in the sense that most of the bias is corrected for) depends on $\rho^{0}$ : it is $M=1$ for $\rho=0, M=2$ for $\rho=0.3, M=3$ and $\rho=0.6$, and $M=5$ for $\rho=0.9$. Choosing too large or too small a bandwidth results in a smaller fraction of the bias to be corrected. Table 4 also reports the properties of the BC-FLS estimator for different values of $\rho^{0}$, $T$, and $M$. It shows the effect of the bandwidth choice on the standard deviation of the BC-FLS estimator is relatively small at $T=40$, but is more pronounced at $T=20$. The issue of optimal bandwidth choice is therefore an important topic for future research. In the simulation results presented here, we tried to choose reasonable values for $M$, but made no attempt to optimize the bandwidth.

In our setup, we have $\left\|\lambda^{0} f^{0 \prime}\right\| \approx \sqrt{2 N T} \sigma_{f}$ and $\|e\| \approx \sqrt{N}+\sqrt{T}{ }^{19}$ Assumptions 1 and 3 imply $\left\|\lambda^{0} f^{0 \prime}\right\| \gg\|e\|$ asymptotically. We can therefore only be sure our asymptotic results for the FLS estimator distribution are a good approximation of the finite sample properties if $\left\|\lambda^{0} f^{0 \prime}\right\| \gtrsim\|e\|$, that is, if $\sqrt{2 N T} \sigma_{f} \gtrsim \sqrt{N}+\sqrt{T}$. To explore this further, we present in Table 5 simulation results for $N=100, T=20, \rho^{0}=0.6$, and different values of $\rho_{f}$ and $\sigma_{f}$. For $\sigma_{f}=0$, we have $0=\left\|\lambda^{0} f^{0 \prime}\right\| \ll\|e\|$, and this case is equivalent to $R=0$ (no factor at all). In this case, the OLS estimator estimates the true model and is almost unbiased, and correspondingly, the FLS estimator and the bias-corrected FLS estimator perform worse than OLS in finite samples (though we expect all three estimators are asymptotically equivalent), but the bias-corrected FLS estimator has a lower bias and a lower variance than the non-corrected FLS estimator. 


\section{Dynamic Panel With Interactive Effects}

The case $\sigma_{f}=0.2$ corresponds to $\left\|\lambda^{0} f^{0 \prime}\right\| \approx\|e\|$, and one finds the bias and the variance of the OLS estimator and of the FLS estimator are of comparable size. However, the bias-corrected FLS estimator already has much smaller bias and a bit smaller variance in this case. Finally, in the case $\sigma_{f}=0.5$, we have $\left\|\lambda^{0} f^{0 \prime}\right\|>\|e\|$, and we expect our asymptotic results to be a good approximation of this situation. Indeed, one finds that for $\sigma_{f}=0.5$, the OLS estimator is heavily biased and very inefficient compared to the FLS estimator, whereas the bias-corrected FLS estimator performs even better in terms of bias and variance.

In Table 6, we present simulation results for the size of the various tests discussed in the last section when testing the null hypothesis $H_{0}: \rho=\rho^{0}$. We choose a nominal size of $5 \%$, $\rho_{f}=0.5, \sigma_{f}=0.5$, and different values for $\rho^{0}, N$, and $T$. In all cases, the size distortions of the uncorrected Wald, LR, and LM test are rather large, and the size distortion of these tests do not vanish as $N$ and $T$ increase: the size for $N=100$ and $T=20$ is about the same as for $N=400$ and $T=80$, and the size for $N=400$ and $T=20$ is about the same as for $N=1600$ and $T=80$. By contrast, the size distortions for the bias-corrected Wald, LR, and LM test are much smaller, and tend toward zero (i.e., the size becomes closer to $5 \%$ ) as $N, T$ increase, holding the ratio $N / T$ constant. For fixed $T$, an increase in $N$ results in a larger size distortion, whereas for fixed $N$, an increase in $T$ results in a smaller size distortion (both for the non-corrected and for the bias-corrected tests).

In Table 7 and 8, we present the power and the size-corrected power when testing the left-sided alternative $H_{a}^{\text {left }}: \rho=\rho^{0}-(N T)^{-1 / 2}$ and the right-sided alternative $H_{a}^{\text {right }}: \rho=$ $\rho^{0}+(N T)^{-1 / 2}$. The model specifications are the same as for the size results in Table 4. Because both the FLS estimator and the bias-corrected FLS estimator for $\rho$ have a negative bias, one finds the power for the left-sided alternative to be much smaller than the power for the rightsided alternative. For the uncorrected tests, this effect can be extreme and the size-corrected power of these tests for the left-sided alternative is below $2 \%$ in all cases and does not improve as $N$ and $T$ become large, holding $N / T$ fixed. By contrast, the power for the bias-corrected tests becomes more symmetric as $N$ and $T$ become large, and the size-corrected power for the left-sided alternative is much larger than for the uncorrected tests, whereas the size-corrected power for the right-sided alternative is about the same.

\section{Conclusions}

This paper studies the least squares estimator for dynamic linear panel regression models with interactive fixed effects. We provide conditions under which the estimator is consistent, allowing 


\section{Dynamic Panel With Interactive Effects}

for predetermined regressors and for a general combination of "low-rank" and "high-rank" regressors. We then show how a quadratic approximation of the profile objective function $L_{N T}(\beta)$ can be used to derive the first-order asymptotic theory of the LS estimator of $\beta$ under the alternative asymptotic $N, T \rightarrow \infty$. We find the asymptotic distribution of the LS estimator can be asymptotically biased (i) because of weak exogeneity of the regressors and (ii) because of heteroscedasticity (and correlation) of the idiosyncratic errors $e_{i t}$. Consistent estimators for the asymptotic covariance matrix and for the asymptotic bias of the LS estimator are provided, and thus a bias-corrected LS estimator is given. We furthermore study the asymptotic distributions of the Wald, LR, and LM test statistics for testing a general linear hypothesis on $\beta$. The uncorrected test statistics are not asymptotically chi-square because of the asymptotic bias of the score and of the LS estimator, but bias-corrected test statistics that are asymptotically chisquare distributed can be constructed. We also discussed a possible extension of the estimation procedure to the case of endogeneous regressors. The findings of our Monte Carlo simulations show our asymptotic results on the distribution of the (bias-corrected) LS estimator and of the (bias-corrected) test statistics provide a good approximation of their finite sample properties. Although the bias-corrected LS estimator has a non-zero bias in finite samples, this bias is much smaller than that of the LS estimator. Analogously, the size distortions and power asymmetries of the bias-corrected Wald, LR, and LM test are much smaller than for the non-bias-corrected versions.

\section{Appendix}

\section{A Proof of Consistency (Theorem 3.1)}

The following theorem is useful for the consistency proof and beyond.

Lemma A.1. Let $N, T, R, R_{1}$, and $R_{2}$ be positive integers such that $R \leq N, R \leq T$, and $R=R_{1}+R_{2}$. Let $Z$ be an $N \times T$ matrix, $\lambda$ be an $N \times R$, $f$ be a $T \times R$ matrix, $\widetilde{\lambda}$ be an $N \times R_{1}$ matrix, and $\tilde{f}$ be a $T \times R_{2}$ matrix. Then the following six expressions (that are functions of $Z$ only) are equivalent:

$$
\begin{gathered}
\min _{f, \lambda} \operatorname{Tr}\left[\left(Z-\lambda f^{\prime}\right)\left(Z^{\prime}-f \lambda^{\prime}\right)\right]=\min _{f} \operatorname{Tr}\left(Z M_{f} Z^{\prime}\right)=\min _{\lambda} \operatorname{Tr}\left(Z^{\prime} M_{\lambda} Z\right) \\
=\min _{\tilde{\lambda}, \tilde{f}} \operatorname{Tr}\left(M_{\tilde{\lambda}} Z M_{\widetilde{f}} Z^{\prime}\right)=\sum_{i=R+1}^{T} \mu_{i}\left(Z^{\prime} Z\right)=\sum_{i=R+1}^{N} \mu_{i}\left(Z Z^{\prime}\right) .
\end{gathered}
$$




\section{Dynamic Panel with Interactive Effects}

In the above minimization problems, we do not have to restrict the matrices $\lambda, f, \widetilde{\lambda}$, and $\tilde{f}$ to be of full rank. If, for example, $\lambda$ is not of full rank, the generalized inverse $\left(\lambda^{\prime} \lambda\right)^{\dagger}$ is still well defined, and the projector $M_{\lambda}$ still satisfies $M_{\lambda} \lambda=0$ and $\operatorname{rank}\left(M_{\lambda}\right)=N-\operatorname{rank}(\lambda)$. If $\operatorname{rank}(Z) \geq R$, the optimal $\lambda, f, \widetilde{\lambda}$, and $\widetilde{f}$ always have full rank.

Lemma A.1 shows the equivalence of the three different versions of the profile objective function in equation (4). It also considers minimization of $\operatorname{Tr}\left(M_{\widetilde{\lambda}} Z M_{\widetilde{f}} Z^{\prime}\right)$ over $\widetilde{\lambda}$ and $\widetilde{f}$, which will be used in the consistency proof below. The proof of the theorem is given in the supplementary material. The following lemma is due to Bai (2009).

Lemma A.2. Under the assumptions of Theorem 3.1 we have

$$
\sup _{f}\left|\frac{\operatorname{Tr}\left(X_{k} M_{f} e^{\prime}\right)}{N T}\right|=o_{p}(1), \quad \sup _{f}\left|\frac{\operatorname{Tr}\left(\lambda^{0} f^{0 \prime} M_{f} e^{\prime}\right)}{N T}\right|=o_{p}(1), \quad \sup _{f}\left|\frac{\operatorname{Tr}\left(e P_{f} e^{\prime}\right)}{N T}\right|=o_{p}(1),
$$

where the parameters $f$ are $T \times R$ matrices with $\operatorname{rank}(f)=R$.

Proof. By Assumption 2, we know the first equation in Lemma A.2 is satisfied when replacing $M_{f}$ by the identity matrix. So we are left to show $\max _{f}\left|\frac{1}{N T} \operatorname{Tr}\left(\Xi e^{\prime}\right)\right|=o_{p}(1)$, where $\Xi$ is either $X_{k} P_{f}, \lambda^{0} f^{0 \prime} M_{f}$, or $e P_{f}$. In all three cases, we have $\|\Xi\| / \sqrt{N T}=\mathcal{O}_{p}(1)$ by Assumption 1,3 , and 4 , respectively, and we have $\operatorname{rank}(\Xi) \leq R$. We therefore find ${ }^{20}$

$$
\sup _{f}\left|\frac{1}{N T} \operatorname{Tr}\left(\Xi P_{f} e^{\prime}\right)\right| \leq R \frac{\|e\|}{\sqrt{N T}} \frac{\|\Xi\|}{\sqrt{N T}}=o_{p}(1) .
$$

Proof of Theorem 3.1. For the second version of the profile objective function in equation (4), we write $L_{N T}(\beta)=\min _{f} S_{N T}(\beta, f)$, where

$$
S_{N T}(\beta, f)=\frac{1}{N T} \operatorname{Tr}\left[\left(\lambda^{0} f^{0 \prime}+\sum_{k=1}^{K}\left(\beta_{k}^{0}-\beta_{k}\right) X_{k}+e\right) M_{f}\left(\lambda^{0} f^{0 \prime}+\sum_{k=1}^{K}\left(\beta_{k}^{0}-\beta_{k}\right) X_{k}+e\right)^{\prime}\right] .
$$

We have $S_{N T}\left(\beta^{0}, f^{0}\right)=\frac{1}{N T} \operatorname{Tr}\left(e M_{f^{0}} e^{\prime}\right)$. Using Lemma (A.2), we find

$$
\begin{aligned}
S_{N T}(\beta, f) & =S_{N T}\left(\beta^{0}, f^{0}\right)+\widetilde{S}_{N T}(\beta, f) \\
& +\frac{2}{N T} \operatorname{Tr}\left[\left(\lambda^{0} f^{0 \prime}+\sum_{k=1}^{K}\left(\beta_{k}^{0}-\beta_{k}\right) X_{k}\right) M_{f} e^{\prime}\right]+\frac{1}{N T} \operatorname{Tr}\left(e\left(P_{f^{0}}-P_{f}\right) e^{\prime}\right) \\
= & S_{N T}\left(\beta^{0}, f^{0}\right)+\widetilde{S}_{N T}(\beta, f)+o_{p}\left(\left\|\beta-\beta^{0}\right\|\right)+o_{p}(1)
\end{aligned}
$$

where we defined

$$
\widetilde{S}_{N T}(\beta, f)=\frac{1}{N T} \operatorname{Tr}\left[\left(\lambda^{0} f^{0 \prime}+\sum_{k=1}^{K}\left(\beta_{k}^{0}-\beta_{k}\right) X_{k}\right) M_{f}\left(\lambda^{0} f^{0 \prime}+\sum_{k=1}^{K}\left(\beta_{k}^{0}-\beta_{k}\right) X_{k}\right)^{\prime}\right] .
$$




\section{Dynamic Panel with Interactive Effects}

Up to this point, the consistency proof is almost equivalent to the one given in Bai (2009), but the remainder of the proof differs from Bai, because we allow for more general low-rank regressors, and because we allow for high-rank and low-rank regressors simultaneously. We split $\widetilde{S}_{N T}(\beta, f)=\widetilde{S}_{N T}^{(1)}(\beta, f)+\widetilde{S}_{N T}^{(2)}(\beta, f)$, where

$$
\begin{aligned}
\widetilde{S}_{N T}^{(1)}(\beta, f) & =\frac{1}{N T} \operatorname{Tr}\left[\left(\lambda^{0} f^{0 \prime}+\sum_{k=1}^{K}\left(\beta_{k}^{0}-\beta_{k}\right) X_{k}\right) M_{f}\left(\lambda^{0} f^{0 \prime}+\sum_{k=1}^{K}\left(\beta_{k}^{0}-\beta_{k}\right) X_{k}\right)^{\prime} M_{\left(\lambda^{0}, w\right)}\right] \\
& =\frac{1}{N T} \operatorname{Tr}\left[\left(\sum_{m=K_{1}+1}^{K}\left(\beta_{m}^{0}-\beta_{m}\right) X_{m}\right) M_{f}\left(\sum_{m=K_{1}+1}^{K}\left(\beta_{m}^{0}-\beta_{m}\right) X_{m}\right)^{\prime} M_{\left(\lambda^{0}, w\right)}\right], \\
\widetilde{S}_{N T}^{(2)}(\beta, f) & =\frac{1}{N T} \operatorname{Tr}\left[\left(\lambda^{0} f^{0 \prime}+\sum_{k=1}^{K}\left(\beta_{k}^{0}-\beta_{k}\right) X_{k}\right) M_{f}\left(\lambda^{0} f^{0 \prime}+\sum_{k=1}^{K}\left(\beta_{k}^{0}-\beta_{k}\right) X_{k}\right)^{\prime} P_{\left(\lambda^{0}, w\right)}\right],
\end{aligned}
$$

and $\left(\lambda^{0}, w\right)$ is the $N \times\left(R+K_{1}\right)$ matrix that is composed out of $\lambda^{0}$ and the $N \times K_{1}$ matrix $w$ defined in Assumption 4. For $\widetilde{S}_{N T}^{(1)}(\beta, f)$, we can apply Lemma A.1 with $\widetilde{f}=f$ and $\widetilde{\lambda}=\left(\lambda^{0}, w\right)$ (the $R$ in the theorem is now $2 R+K_{1}$ ) to find

$$
\begin{aligned}
\widetilde{S}_{N T}^{(1)}(\beta, f) & \geq \frac{1}{N T} \sum_{i=2 R+K_{1}+1}^{N} \mu_{i}\left[\left(\sum_{m=K_{1}+1}^{K}\left(\beta_{m}^{0}-\beta_{m}\right) X_{m}\right)\left(\sum_{m=K_{1}+1}^{K}\left(\beta_{m}^{0}-\beta_{m}\right) X_{m}\right)^{\prime}\right] \\
& \geq b\left\|\beta^{\text {high }}-\beta^{0, \text { high }}\right\|^{2}, \quad \text { wpa1, }
\end{aligned}
$$

where in the last step, we used the existence of a constant $b>0$ guaranteed by Assumption 4(ii)(a), and we introduced $\beta^{\text {high }}=\left(\beta_{K_{1}+1}, \ldots, \beta_{K}\right)^{\prime}$, which refers to the $K_{2} \times 1$ parameter vector corresponding to the high-rank regressors. Similarly, we define $\beta^{\text {low }}=\left(\beta_{1}, \ldots, \beta_{K_{1}}\right)^{\prime}$ for the $K_{1} \times 1$ parameter vector of low-rank regressors.

Using $P_{\left(\lambda^{0}, w\right)}=P_{\left(\lambda^{0}, w\right)} P_{\left(\lambda^{0}, w\right)}$ and the cyclicality of the trace, we see $\widetilde{S}_{N T}^{(2)}(\beta, f)$ can be written as the trace of a positive definite matrix, and therefore $\widetilde{S}_{N T}^{(2)}(\beta, f) \geq 0$. Note also that we can choose $\beta=\beta^{0}$ and $f=f^{0}$ in the minimization problem over $S_{N T}(\beta, f)$; that is, the optimal $\beta=\widehat{\beta}$ and $f=\widehat{f}$ must satisfy $S_{N T}(\widehat{\beta}, \widehat{f}) \leq S_{N T}\left(\beta^{0}, f^{0}\right)$. Using this result, equation (12), $\widetilde{S}_{N T}^{(2)}(\beta, f) \geq 0$, and the bound in $(13)$, we find

$$
0 \geq b\left\|\widehat{\beta}^{\text {high }}-\beta^{0, \text { high }}\right\|^{2}+o_{p}\left(\left\|\widehat{\beta}^{\text {high }}-\beta^{0, \text { high }}\right\|\right)+o_{p}\left(\left\|\widehat{\beta}^{\text {low }}-\beta^{0, \text { low }}\right\|\right)+o_{p}(1) .
$$

Because we assume $\widehat{\beta}^{\text {low }}$ is bounded, the last equation implies $\left\|\widehat{\beta}^{\text {high }}-\beta^{0, \text { high }}\right\|=o_{p}(1)$; that is, $\widehat{\beta}^{\text {high }}$ is consistent. What is left to show is that $\widehat{\beta}^{\text {low }}$ is consistent, too. In the supplementary material, we show Assumption 4(ii)(b) guarantees that finite positive constants $a_{0}, a_{1}, a_{2}, a_{3}$, 
and $a_{4}$ exist such that

$$
\begin{aligned}
\widetilde{S}_{N T}^{(2)}(\beta, f) \geq \frac{a_{0}\left\|\beta^{\text {low }}-\beta^{0, \text { low }}\right\|^{2}}{\left\|\beta^{\text {low }}-\beta^{0, \text { low }}\right\|^{2}+a_{1}\left\|\beta^{\text {low }}-\beta^{0, \text { low }}\right\|+a_{2}} \\
\quad-a_{3}\left\|\beta^{\text {high }}-\beta^{0, \text { high }}\right\|-a_{4}\left\|\beta^{\text {high }}-\beta^{0, \text { high }}\right\|\left\|\beta^{\text {low }}-\beta^{0, \text { low }}\right\|, \quad \text { wpa1. }
\end{aligned}
$$

Using consistency of $\widehat{\beta}^{\text {high }}$ and again boundedness of $\beta^{\text {low }}$, the previous inequality implies $a>0$

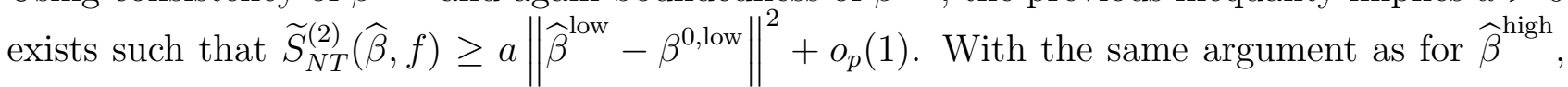
we therefore find $\left\|\widehat{\beta}^{\text {low }}-\beta^{0 \text {,low }}\right\|=o_{p}(1)$; that is, $\widehat{\beta}^{\text {low }}$ is consistent.

\section{B Proof of Limiting Distribution (Theorem 4.3)}

Theorem 4.1 is from Moon and Weidner (2015), and the proof can be found there. Note Assumption 4(i) implies $\left\|X_{k}\right\|=\mathcal{O}_{p}(\sqrt{N T})$, which we assume in Moon and Weidner (2015). There, we also assume that $\|e\|=\mathcal{O}_{p}(\sqrt{\max (N, T)})=\mathcal{O}_{p}(\sqrt{N})$, whereas in the current paper we assume $\|e\|=o_{p}\left(\left\|N^{2 / 3}\right\|\right)$. It is, however, straightforward to verify that the proof of Theorem 4.1 is also valid under this weaker assumption.

Moon and Weidner (2015) also includes the proof of Corollary 4.2. The proof requires

consistency of $\widehat{\beta}$, which in the current paper is derived under weaker assumptions than in Moon and Weidner (2015), where no low-rank regressors are considered. Corollary 4.2 is therefore stated under weaker assumptions here, but the proof is unchanged. In the supplementary material, we show the assumptions of Corollary 4.2 already guarantee $W_{N T}$ does not become singular as $N, T \rightarrow \infty$.

For each $k=1, \ldots, K$, we define the $N \times T$ matrices $\bar{X}_{k}, \widetilde{X}_{k}$, and $\mathfrak{X}_{k}$ as follows:

$$
\bar{X}_{k}=\mathbb{E}\left(X_{k} \mid \mathcal{C}\right), \quad \tilde{X}_{k}=X_{k}-\mathbb{E}\left(X_{k} \mid \mathcal{C}\right), \quad \mathfrak{X}_{k}=M_{\lambda^{0}} \bar{X}_{k} M_{f^{0}}+\tilde{X}_{k} .
$$

Note the difference between $\mathfrak{X}_{k}$ and $\mathcal{X}_{k}=M_{\lambda^{0}} X_{k} M_{f^{0}}$, which was defined in Assumption 6 . In particular, conditional on $\mathcal{C}$, the elements $\mathfrak{X}_{k, i t}$ of $\mathfrak{X}_{k}$ are contemporaneously uncorrelated with the error term $e_{i t}$, although the same is not true for $\mathcal{X}_{k}$.

To present the proof of Theorem 4.3, it is convenient to first state two technical lemmas. 


\section{Dynamic Panel With Interactive Effects}

Lemma B.1. Under the assumptions of Theorem 4.3, we have

$(g)$

$$
\begin{aligned}
& \frac{1}{\sqrt{N T}} \operatorname{Tr}\left(P_{f^{0}} e^{\prime} P_{\lambda^{0}} \widetilde{X}_{k}\right)=o_{p}(1), \\
& \frac{1}{\sqrt{N T}} \operatorname{Tr}\left(P_{\lambda^{0}} \text { e } \tilde{X}_{k}^{\prime}\right)=o_{p}(1) \\
& \frac{1}{\sqrt{N T}} \operatorname{Tr}\left\{P_{f^{0}}\left[e^{\prime} \tilde{X}_{k}-\mathbb{E}\left(e^{\prime} \tilde{X}_{k} \mid \mathcal{C}\right)\right]\right\}=o_{p}(1) \\
& \frac{1}{\sqrt{N T}} \operatorname{Tr}\left(e P_{f^{0}} e^{\prime} M_{\lambda^{0}} X_{k} f^{0}\left(f^{0 \prime} f^{0}\right)^{-1}\left(\lambda^{0 \prime} \lambda^{0}\right)^{-1} \lambda^{0 \prime}\right)=o_{p}(1) \text {, } \\
& \frac{1}{\sqrt{N T}} \operatorname{Tr}\left(e^{\prime} P_{\lambda^{0}} \text { e } M_{f^{0}} X_{k}^{\prime} \lambda^{0}\left(\lambda^{0 \prime} \lambda^{0}\right)^{-1}\left(f^{0 \prime} f^{0}\right)^{-1} f^{0 \prime}\right)=o_{p}(1), \\
& \frac{1}{\sqrt{N T}} \operatorname{Tr}\left(e^{\prime} M_{\lambda^{0}} X_{k} M_{f^{0}} e^{\prime} \lambda^{0}\left(\lambda^{0 \prime} \lambda^{0}\right)^{-1}\left(f^{0 \prime} f^{0}\right)^{-1} f^{0 \prime}\right)=o_{p}(1), \\
& \frac{1}{\sqrt{N T}} \operatorname{Tr}\left\{\left[e e^{\prime}-\mathbb{E}\left(e e^{\prime} \mid \mathcal{C}\right)\right] M_{\lambda^{0}} X_{k} f^{0}\left(f^{0 \prime} f^{0}\right)^{-1}\left(\lambda^{0 \prime} \lambda^{0}\right)^{-1} \lambda^{0 \prime}\right\}=o_{p}(1), \\
& \frac{1}{\sqrt{N T}} \operatorname{Tr}\left\{\left[e^{\prime} e-\mathbb{E}\left(e^{\prime} e \mid \mathcal{C}\right)\right] M_{f^{0}} X_{k}^{\prime} \lambda^{0}\left(\lambda^{0 \prime} \lambda^{0}\right)^{-1}\left(f^{0 \prime} f^{0}\right)^{-1} f^{0 \prime}\right\}=o_{p}(1), \\
& \frac{1}{N T} \sum_{i=1}^{N} \sum_{t=1}^{T}\left[e_{i t}^{2} \mathfrak{X}_{i t} \mathfrak{X}_{i t}^{\prime}-\mathbb{E}\left(e_{i t}^{2} \mathfrak{X}_{i t} \mathfrak{X}_{i t}^{\prime} \mid \mathcal{C}\right)\right]=o_{p}(1), \\
& \frac{1}{N T} \sum_{i=1}^{N} \sum_{t=1}^{T} e_{i t}^{2}\left(\mathfrak{X}_{i t} \mathfrak{X}_{i t}^{\prime}-\mathcal{X}_{i t} \mathcal{X}_{i t}^{\prime}\right)=o_{p}(1) .
\end{aligned}
$$

Lemma B.2. Under the assumptions of Theorem 4.3, we have

$$
\frac{1}{\sqrt{N T}} \sum_{i=1}^{N} \sum_{t=1}^{T} e_{i t} \mathfrak{X}_{i t} \underset{d}{\rightarrow} \mathcal{N}(0, \Omega) .
$$

The proofs of Lemma B.1 and Lemma B.2 are provided in the supplementary material. We briefly want to discuss why the asymptotic variance-covariance matrix in Lemma B.2 turns out to be $\Omega$. Note that because $e_{i t} \mathfrak{X}_{i t}$ is mean zero and uncorrelated across both $i$ and $t$, conditional on $\mathcal{C}$, we have

$$
\begin{aligned}
\operatorname{Var}\left(\frac{1}{\sqrt{N T}} \sum_{i=1}^{N} \sum_{t=1}^{T} e_{i t} \mathfrak{X}_{i t} \mid \mathcal{C}\right) & =\frac{1}{N T} \sum_{i=1}^{N} \sum_{t=1}^{T} \mathbb{E}\left(e_{i t}^{2} \mathfrak{X}_{i t} \mathfrak{X}_{i t}^{\prime} \mid \mathcal{C}\right) \\
& =\frac{1}{N T} \sum_{i=1}^{N} \sum_{t=1}^{T} e_{i t}^{2} \mathfrak{X}_{i t} \mathfrak{X}_{i t}^{\prime}+o_{p}(1) \\
& =\frac{1}{N T} \sum_{i=1}^{N} \sum_{t=1}^{T} e_{i t}^{2} \mathcal{X}_{i t} \mathcal{X}_{i t}^{\prime}+o_{p}(1) \\
& =\Omega+o_{p}(1),
\end{aligned}
$$


where we also used part (i) of Lemma B.1 for the second equality and part (j) of Lemma B.1 for the third equality, and the definition of $\Omega$ in Assumptions 6 in the last step.

Using those lemmas, we can now prove the theorem on the limiting distribution of $\widehat{\beta}$ in the main text.

Proof of Theorem 4.3. Assumption 5 implies $\|e\|=\mathcal{O}_{p}\left(N^{1 / 2}\right)$ as $N$ and $T$ grow at the same rate, as discussed in section S.2 of the supplementary material; that is, Assumption $3^{*}$ is satisfied. We can therefore apply Corollary 4.2 to calculate the limiting distribution of $\widehat{\beta}$. Note that $M_{\lambda^{0}} X_{k} M_{f^{0}}=\mathfrak{X}_{k}-\widetilde{X}_{k} P_{f^{0}}-P_{\lambda^{0}} \tilde{X}_{k}+P_{\lambda^{0}} \tilde{X}_{k} P_{f^{0}}$. Using Lemmas B.1 and B.2 and Assumption 6 , we find

$$
\begin{aligned}
& \frac{1}{\sqrt{N T}} C^{(1)}\left(\lambda^{0}, f^{0}, X_{k}, e\right)=\frac{1}{\sqrt{N T}} \operatorname{Tr}\left(e^{\prime} M_{\lambda^{0}} X_{k} M_{f^{0}}\right) \\
& =\frac{1}{\sqrt{N T}} \operatorname{Tr}\left(e^{\prime} \mathfrak{X}_{k}\right)-\frac{1}{\sqrt{N T}} \operatorname{Tr}\left[P_{f^{0}} \mathbb{E}\left(e^{\prime} \tilde{X}_{k} \mid \mathcal{C}\right)\right] \\
& -\frac{1}{\sqrt{N T}} \operatorname{Tr}\left(e^{\prime} P_{\lambda^{0}} \widetilde{X}_{k}\right)+\frac{1}{\sqrt{N T}} \operatorname{Tr}\left(P_{f^{0}} e^{\prime} P_{\lambda^{0}} \widetilde{X}_{k}\right) \\
& -\frac{1}{\sqrt{N T}} \operatorname{Tr}\left\{P_{f^{0}}\left[e^{\prime} \widetilde{X}_{k}-\mathbb{E}\left(e^{\prime} \widetilde{X}_{k} \mid \mathcal{C}\right)\right]\right\} \\
& =\frac{1}{\sqrt{N T}} \operatorname{Tr}\left(e^{\prime} \mathfrak{X}_{k}\right)-\frac{1}{\sqrt{N T}} \operatorname{Tr}\left[P_{f^{0}} \mathbb{E}\left(e^{\prime} X_{k} \mid \mathcal{C}\right)\right]+o_{p}(1) \text {. } \\
& \underset{d}{\rightarrow} \mathcal{N}\left(-\kappa B_{1}, \Omega\right)
\end{aligned}
$$




\section{Dynamic Panel With Interactive Effects}

where we also used that $\mathbb{E}\left(e^{\prime} \tilde{X}_{k} \mid \mathcal{C}\right)=\mathbb{E}\left(e^{\prime} X_{k} \mid \mathcal{C}\right)$. Using Lemma B.1, we also find

$$
\begin{aligned}
& \frac{1}{\sqrt{N T}} C^{(2)}\left(\lambda^{0}, f^{0}, X_{k}, e\right)=-\frac{1}{\sqrt{N T}}\left[\operatorname{Tr}\left(e M_{f^{0}} e^{\prime} M_{\lambda^{0}} X_{k} f^{0}\left(f^{0 \prime} f^{0}\right)^{-1}\left(\lambda^{0 \prime} \lambda^{0}\right)^{-1} \lambda^{0 \prime}\right)\right. \\
& +\operatorname{Tr}\left(e^{\prime} M_{\lambda^{0}} e M_{f^{0}} X_{k}^{\prime} \lambda^{0}\left(\lambda^{0 \prime} \lambda^{0}\right)^{-1}\left(f^{0 \prime} f^{0}\right)^{-1} f^{0 \prime}\right) \\
& \left.+\operatorname{Tr}\left(e^{\prime} M_{\lambda^{0}} X_{k} M_{f^{0}} e^{\prime} \lambda^{0}\left(\lambda^{0 \prime} \lambda^{0}\right)^{-1}\left(f^{0 \prime} f^{0}\right)^{-1} f^{0 \prime}\right)\right] \\
& =\frac{1}{\sqrt{N T}} \operatorname{Tr}\left(e P_{f^{0}} e^{\prime} M_{\lambda^{0}} X_{k} f^{0}\left(f^{0 \prime} f^{0}\right)^{-1}\left(\lambda^{0 \prime} \lambda^{0}\right)^{-1} \lambda^{0 \prime}\right) \\
& -\frac{1}{\sqrt{N T}} \operatorname{Tr}\left\{\left[e e^{\prime}-\mathbb{E}\left(e e^{\prime} \mid \mathcal{C}\right)\right] M_{\lambda^{0}} X_{k} f^{0}\left(f^{0 \prime} f^{0}\right)^{-1}\left(\lambda^{0 \prime} \lambda^{0}\right)^{-1} \lambda^{0 \prime}\right\} \\
& -\frac{1}{\sqrt{N T}} \operatorname{Tr}\left[\mathbb{E}\left(e e^{\prime} \mid \mathcal{C}\right) M_{\lambda^{0}} X_{k} f^{0}\left(f^{0 \prime} f^{0}\right)^{-1}\left(\lambda^{0 \prime} \lambda^{0}\right)^{-1} \lambda^{0 \prime}\right] \\
& +\frac{1}{\sqrt{N T}} \operatorname{Tr}\left(e^{\prime} P_{\lambda^{0}} \text { e } M_{f^{0}} X_{k}^{\prime} \lambda^{0}\left(\lambda^{0 \prime} \lambda^{0}\right)^{-1}\left(f^{0 \prime} f^{0}\right)^{-1} f^{0 \prime}\right) \\
& -\frac{1}{\sqrt{N T}} \operatorname{Tr}\left\{\left[e^{\prime} e-\mathbb{E}\left(e^{\prime} e \mid \mathcal{C}\right)\right] M_{f^{0}} X_{k}^{\prime} \lambda^{0}\left(\lambda^{0 \prime} \lambda^{0}\right)^{-1}\left(f^{0 \prime} f^{0}\right)^{-1} f^{0 \prime}\right\} \\
& -\frac{1}{\sqrt{N T}} \operatorname{Tr}\left[\mathbb{E}\left(e^{\prime} e \mid \mathcal{C}\right) M_{f^{0}} X_{k}^{\prime} \lambda^{0}\left(\lambda^{0 \prime} \lambda^{0}\right)^{-1}\left(f^{0 \prime} f^{0}\right)^{-1} f^{0 \prime}\right] \\
& +\frac{1}{\sqrt{N T}} \operatorname{Tr}\left(e^{\prime} M_{\lambda^{0}} X_{k} M_{f^{0}} e^{\prime} \lambda^{0}\left(\lambda^{0 \prime} \lambda^{0}\right)^{-1}\left(f^{0 \prime} f^{0}\right)^{-1} f^{0 \prime}\right) \\
& =-\frac{1}{\sqrt{N T}} \operatorname{Tr}\left[\mathbb{E}\left(e e^{\prime} \mid \mathcal{C}\right) M_{\lambda^{0}} X_{k} f^{0}\left(f^{0 \prime} f^{0}\right)^{-1}\left(\lambda^{0 \prime} \lambda^{0}\right)^{-1} \lambda^{0 \prime}\right] \\
& -\frac{1}{\sqrt{N T}} \operatorname{Tr}\left[\mathbb{E}\left(e^{\prime} e \mid \mathcal{C}\right) M_{f^{0}} X_{k}^{\prime} \lambda^{0}\left(\lambda^{0 \prime} \lambda^{0}\right)^{-1}\left(f^{0 \prime} f^{0}\right)^{-1} f^{0 \prime}\right]+o_{p}(1), \\
& =-\kappa^{-1} B_{2}-\kappa B_{3}+o_{p}(1) \text {. }
\end{aligned}
$$

Combining these results, we obtain

$$
\begin{aligned}
\sqrt{N T}\left(\widehat{\beta}-\beta^{0}\right) & =W_{N T}^{-1} \frac{1}{\sqrt{N T}} C_{N T} \\
& \rightarrow \mathcal{N}\left(-W^{-1}\left(\kappa B_{1}+\kappa^{-1} B_{2}+\kappa B_{3}\right), W^{-1} \Omega W^{-1}\right),
\end{aligned}
$$

which is what we wanted to show.

\section{Notes}

${ }^{1}$ See, e.g., Chamberlain and Rothschild (1983), Ross (1976), and Fama and French (1993) for asset pricing; Stock and Watson (2002) and Bai and Ng (2006) for forecasting; Bernanke, Boivin, and Eliasz (2005) for empirical macro; and Holtz-Eakin, Newey, and Rosen (1988) for empirical labor economics. 


\section{Dynamic Panel With Interactive Effects}

${ }^{2}$ The theory of the CCE estimator was further developed in, e.g., Harding and Lamarche (2009; 2011), Kapetanios, Pesaran, and Yamagata (2011), Pesaran and Tosetti (2011), Chudik, Pesaran, and Tosetti (2011), and Chudik and Pesaran (2015).

${ }^{3}$ The LS estimator is sometimes called "concentrated" least squares estimator in the literature, and in an earlier version of the paper, we referred to it as the "Gaussian Quasi Maximum Likelihood Estimator", because LS estimation is equivalent to maximizing a conditional Gaussian likelihood function.

${ }^{4}$ Hahn and Kuersteiner (2002) introduced the alternative asymptotics to characterize the asymptotic bias due to incidental parameter problems in fixed effect dynamic panel data models. See also Arellano and Hahn (2007) and Moon, Perron, and Phillips (2014) and references therein.

${ }^{5}$ The "likelihood ratio" and the score used in the tests are based on the LS objective function, which can be interpreted as the (misspecified) conditional Gaussian likelihood function.

${ }^{6}$ Another type of widely studied tests in the interactive fixed effect panel literature are panel unit root tests, e.g., Bai and Ng (2004), Moon and Perron (2004), and Phillips and Sul (2003).

${ }^{7}$ In Moon and Weidner (2015) we do not consider low-rank regressors or testing problems, and we impose more restrictive assumptions on the error term of the model implying that some leading bias terms of the LS estimator are not present.

${ }^{8}$ Lee, Moon, and Weidner (2012) also apply the MSW estimation method to estimate a simple dynamic panel regression with interactive fixed effect and classical measurement errors.

${ }^{9}$ To remove this restriction, one could estimate $R$ consistently in the presence of the regressors. In the literature so far, however, consistent estimation procedures for $R$ are established mostly in pure factor models (e.g., Bai and Ng (2002), Onatski (2010) and Harding (2007)). Alternatively, one could rely on Moon and Weidner (2015) who consider a regression model with interactive fixed effects when only an upper bound on the number of factors is known — but extending those results to the more general setup considered here is mathematically challenging.

${ }^{10}$ If we have low-rank regressors with rank larger than one, then we write $X_{l}=w_{l} v_{l}^{\prime}$, where $w_{l}$ is an $N \times \operatorname{rank}\left(X_{l}\right)$ matrix and $v_{l}$ is a $T \times \operatorname{rank}\left(X_{l}\right)$ matrix, and we define $w=\left(w_{1}, \ldots, w_{K_{1}}\right)$ as a $N \times \sum_{l=1}^{L} \operatorname{rank}\left(X_{l}\right) \operatorname{matrix}$, and $v=\left(v_{1}, \ldots, v_{K_{1}}\right)$ ae a $T \times \sum_{l=1}^{L} \operatorname{rank}\left(X_{l}\right)$ matrix. All our results are then unchanged, as long as $\operatorname{rank}\left(X_{l}\right)$ is a finite constant for all $l=1, \ldots, K_{1}$, and we replace $2 R+K_{1}$ by $2 R+\operatorname{rank}(w)$ in $\operatorname{Assumption} \operatorname{ID}(v)$ and Assumption $4(i i)(a)$.

${ }^{11}$ Note that $\operatorname{rank}\left(\lambda^{0}\right)=R$ if $R$ factors are present. Our identification results are consistent with the possibility that $\operatorname{rank}\left(\lambda^{0}\right)<R$, i.e., that $R$ only represents an upper bound on the number of factors, but later we assume $\operatorname{rank}\left(\lambda^{0}\right)=R$ to show consistency.

${ }^{12}$ We could write $X_{k}^{(N, T)}, e^{(N, T)}, \lambda^{(N, T)}$, and $f^{(N, T)}$, because all these matrices, and even their dimensions, are functions on $N$ and $T$, but we suppress this dependence throughout the paper.

${ }^{13}$ Here and in the following, we write $\sigma(A)$ for the sigma algebra generated by the (collection of) random variable(s) $A$, and we write $\mathcal{A} \vee \mathcal{B}$ for the sigma algebra generated by the unions of all elements in the sigma algebra $\mathcal{A}$ and $\mathcal{B}$, so that in the conditional expectation in Assumption 5(ii), we condition jointly on $\mathcal{C}$ and $\left\{\left(X_{i s}, e_{i, s-1}\right), s \leq t\right\}$.

${ }^{14}$ Assumption 2 and $3^{*}$ are implied by Assumption 5 and therefore need not be explicitly assumed here.

${ }^{15}$ Alternatively, one could use $\widehat{B}(\widetilde{\beta})$ and $\widehat{W}(\widetilde{\beta})$ as estimates for $B$ and $W$, and would obtain the same limiting distribution of $L R_{N T}^{*}$ under the null hypothesis $H_{0}$. These alternative estimators are not consistent if $H_{0}$ is false, 


\section{Dynamic Panel With Interactive Effects}

i.e. the power-properties of the test would be different. The question of which specification should be preferred is left for future research.

${ }^{16}$ The proof of the statement is given in the supplementary material as part of the proof of Theorem 5.2.

${ }^{17}$ Chernazhukov and Hansen (2005) also used a similar method for estimating endogenous quantile regression models.

${ }^{18}$ Here we can either use $\mathbb{B}=(-1,1)$, or $\mathbb{B}=\mathbb{R}$. In the present model, we only have high-rank regressors; i.e., the parameter space need not be bounded to show consistency.

${ }^{19}$ To be precise, we have $\left\|\lambda^{0} f^{0 \prime}\right\| /\left(\sqrt{2 N T} \sigma_{f}\right) \rightarrow_{p} 1$, and $\|e\| /(\sqrt{N}+\sqrt{T}) \rightarrow_{p} 1$.

${ }^{20}$ Here we use $|\operatorname{Tr}(C)| \leq\|C\| \operatorname{rank}(C)$, which holds for all square matrices $C$; see the supplementary material.

\section{References}

Ahn, S. C., Lee, Y. H., and Schmidt, P. (2001). GMM estimation of linear panel data models with time-varying individual effects. Journal of Econometrics, 101(2):219-255.

Andrews, D. W. K. (1999). Estimation when a parameter is on a boundary. Econometrica, 67(6):1341-1384.

Andrews, D. W. K. (2001). Testing when a parameter is on the boundary of the maintained hypothesis. Econometrica, 69(3):683-734.

Arellano, M. and Hahn, J. (2007). Understanding bias in nonlinear panel models: Some recent developments. Econometric Society Monographs, 43:381.

Bai, J. (2009). Panel data models with interactive fixed effects. Econometrica, 77(4):1229-1279.

Bai, J. and Ng, S. (2002). Determining the number of factors in approximate factor models. Econometrica, 70(1):191-221.

Bai, J. and Ng, S. (2004). A panic attack on unit roots and cointegration. Econometrica, $72(4): 1127-1177$.

Bai, J. and Ng, S. (2006). Confidence intervals for diffusion index forecasts and inference for factor-augmented regressions. Econometrica, 74(4):1133-1150.

Bai, Z. D., Silverstein, J. W., and Yin, Y. Q. (1988). A note on the largest eigenvalue of a large dimensional sample covariance matrix. J. Multivar. Anal., 26(2):166-168.

Bernanke, B. S., Boivin, J., and Eliasz, P. (2005). Measuring the effects of monetary policy: A factor-augmented vector autoregressive (favar) approach. The Quarterly Journal of Economics, 120(1):387-422.

Berry, S., Levinsohn, J., and Pakes, A. (1995). Automobile prices in market equilibrium. Econometrica, pages 841-890. 


\section{Dynamic Panel With Interactive Effects}

Chamberlain, G. and Rothschild, M. (1983). Arbitrage, factor structure, and mean-variance analysis on large asset markets. Econometrica, 51(5):1281-1304.

Chernozhukov, V. and Hansen, C. (2005). An iv model of quantile treatment effects. Econometrica, 73(1):245-261.

Chudik, A. and Pesaran, M. H. (2015). Common correlated effects estimation of heterogeneous dynamic panel data models with weakly exogenous regressors. Journal of Econometrics.

Chudik, A., Pesaran, M. H., and Tosetti, E. (2011). Weak and strong cross-section dependence and estimation of large panels. The Econometrics Journal, 14(1):C45-C90.

Dhaene, G. and Jochmans, K. (2015). Split-panel jackknife estimation of fixed-effect models. The Review of Economic Studies.

Fama, E. F. and French, K. R. (1993). Common risk factors in the returns on stocks and bonds. Journal of Financial Economics, 33(1):3-56.

Fernández-Val, I. and Weidner, M. (2013). Individual and time effects in nonlinear panel data models with large N, T. CeMMAP working paper series.

Geman, S. (1980). A limit theorem for the norm of random matrices. Annals of Probability, $8(2): 252-261$.

Gobillon, L. and Magnac, T. (2013). Regional policy evaluation: Interactive fixed effects and synthetic controls. IZA Discussion Paper No. 7493.

Hahn, J. and Kuersteiner, G. (2002). Asymptotically unbiased inference for a dynamic panel model with fixed effects when both "n" and "T" are large. Econometrica, 70(4):1639-1657.

Hahn, J. and Kuersteiner, G. (2011). Bias reduction for dynamic nonlinear panel models with fixed effects. Econometric Theory, 27(06):1152-1191.

Hahn, J. and Newey, W. (2004). Jackknife and analytical bias reduction for nonlinear panel models. Econometrica, 72(4):1295-1319.

Harding, M. (2007). Structural estimation of high-dimensional factor models. unpublished manuscript.

Harding, M. and Lamarche, C. (2009). A quantile regression approach for estimating panel data models using instrumental variables. Economics Letters, 104(3):133-135.

Harding, M. and Lamarche, C. (2011). Least squares estimation of a panel data model with multifactor error structure and endogenous covariates. Economics Letters, 111(3):197-199.

Holtz-Eakin, D., Newey, W., and Rosen, H. S. (1988). Estimating vector autoregressions with panel data. Econometrica, 56(6):1371-95.

Kapetanios, G., Pesaran, M. H., and Yamagata, T. (2011). Panels with non-stationary multifactor error structures. Journal of Econometrics, 160(2):326-348. 


\section{Dynamic Panel With Interactive Effects}

Kato, T. (1980). Perturbation Theory for Linear Operators. Springer-Verlag.

Latala, R. (2005). Some estimates of norms of random matrices. Proc. Amer. Math. Soc., 133:1273-1282.

Lee, N., Moon, H. R., and Weidner, M. (2012). Analysis of interactive fixed effects dynamic linear panel regression with measurement error. Economics Letters, 117(1):239-242.

Moon, H., Shum, M., and Weidner, M. (2012). Interactive fixed effects in the BLP random coefficients demand model. CeMMAP working paper series.

Moon, H. R. and Perron, B. (2004). Testing for a unit root in panels with dynamic factors. Journal of Econometrics, 122(1):81-126.

Moon, H. R. and Weidner, M. (2015). Linear regression for panel with unknown number of factors as interactive fixed effects. Econometrica, 83(4):1543-1579.

Moon, R., Perron, B., and Phillips, P. C. (2014). Incidental parameters and dynamic panel modeling. forthcoming in The Oxford Handbook of Panel Data, Chapter 4.

Nickell, S. (1981). Biases in dynamic models with fixed effects. Econometrica, 49(6):1417-1426.

Onatski, A. (2010). Determining the number of factors from empirical distribution of eigenvalues. The Review of Economics and Statistics, 92(4):1004-1016.

Pesaran, M. H. (2006). Estimation and inference in large heterogeneous panels with a multifactor error structure. Econometrica, 74(4):967-1012.

Pesaran, M. H. and Tosetti, E. (2011). Large panels with common factors and spatial correlation. Journal of Econometrics, 161(2):182-202.

Phillips, P. C. B. and Sul, D. (2003). Dynamic panel estimation and homogeneity testing under cross section dependence. Econometrics Journal, 6(1):217-259.

Ross, S. A. (1976). The arbitrage theory of capital asset pricing. Journal of Economic Theory, 13(3):341-360.

Silverstein, J. W. (1989). On the eigenvectors of large dimensional sample covariance matrices. J. Multivar. Anal., 30(1):1-16.

Stock, J. H. and Watson, M. W. (2002). Forecasting using principal components from a large number of predictors. Journal of the American Statistical Association, 97:1167-1179.

Yin, Y. Q., Bai, Z. D., and Krishnaiah, P. (1988). On the limit of the largest eigenvalue of the large-dimensional sample covariance matrix. Probability Theory Related Fields, 78:509-521. 


\section{Tables with Simulation Results}

Table 1: Simulation results for the $\mathrm{AR}(1)$ model described in the main text with $N=100$, $\rho_{f}=0.5, \sigma_{f}=0.5$, and different values of $T$ (with corresponding bandwidth $M$ ) and true $\operatorname{AR}(1)$ coefficient $\rho^{0}$.

\begin{tabular}{llllllll}
\hline & & \multicolumn{3}{c}{$\rho^{0}=0.3$} & & \multicolumn{3}{c}{$\rho^{0}=0.9$} \\
& & OLS & FLS & BC-FLS & OLS & FLS & BC-FLS \\
\hline$T=5$ & bias & 0.1232 & -0.1419 & -0.0713 & 0.0200 & -0.3686 & -0.2330 \\
$(M=2)$ & std & 0.1444 & 0.1480 & 0.0982 & 0.0723 & 0.1718 & 0.1301 \\
& rmse & 0.1898 & 0.2050 & 0.1213 & 0.0750 & 0.4067 & 0.2669 \\
$T=10$ & bias & 0.1339 & -0.0542 & -0.0201 & 0.0218 & -0.1019 & -0.0623 \\
$(M=3)$ & std & 0.1148 & 0.0596 & 0.0423 & 0.0513 & 0.1094 & 0.0747 \\
& rmse & 0.1764 & 0.0806 & 0.0469 & 0.0557 & 0.1495 & 0.0973 \\
$T=20$ & bias & 0.1441 & -0.0264 & -0.0070 & 0.0254 & -0.0173 & -0.0085 \\
$(M=4)$ & std & 0.0879 & 0.0284 & 0.0240 & 0.0353 & 0.0299 & 0.0219 \\
& rmse & 0.1687 & 0.0388 & 0.0250 & 0.0434 & 0.0345 & 0.0235 \\
$T=40$ & bias & 0.1517 & -0.0130 & -0.0021 & 0.0294 & -0.0057 & -0.0019 \\
$(M=5)$ & std & 0.0657 & 0.0170 & 0.0160 & 0.0250 & 0.0105 & 0.0089 \\
& rmse & 0.1654 & 0.0214 & 0.0161 & 0.0386 & 0.0119 & 0.0091 \\
$T=80$ & bias & 0.1552 & -0.0066 & -0.0007 & 0.0326 & -0.0026 & -0.0006 \\
$(M=6)$ & std & 0.0487 & 0.0112 & 0.0109 & 0.0179 & 0.0056 & 0.0053 \\
& rmse & 0.1627 & 0.0130 & 0.0109 & 0.0372 & 0.0062 & 0.0053 \\
\hline
\end{tabular}




\section{Dynamic Panel with Interactive Effects}

Table 2: Same DGP as Table 1, but misspecification in number of factors $R$ is present. The true number of factors is $R=1$, but the FLS and BC-FLS are calculated with $R=2$.

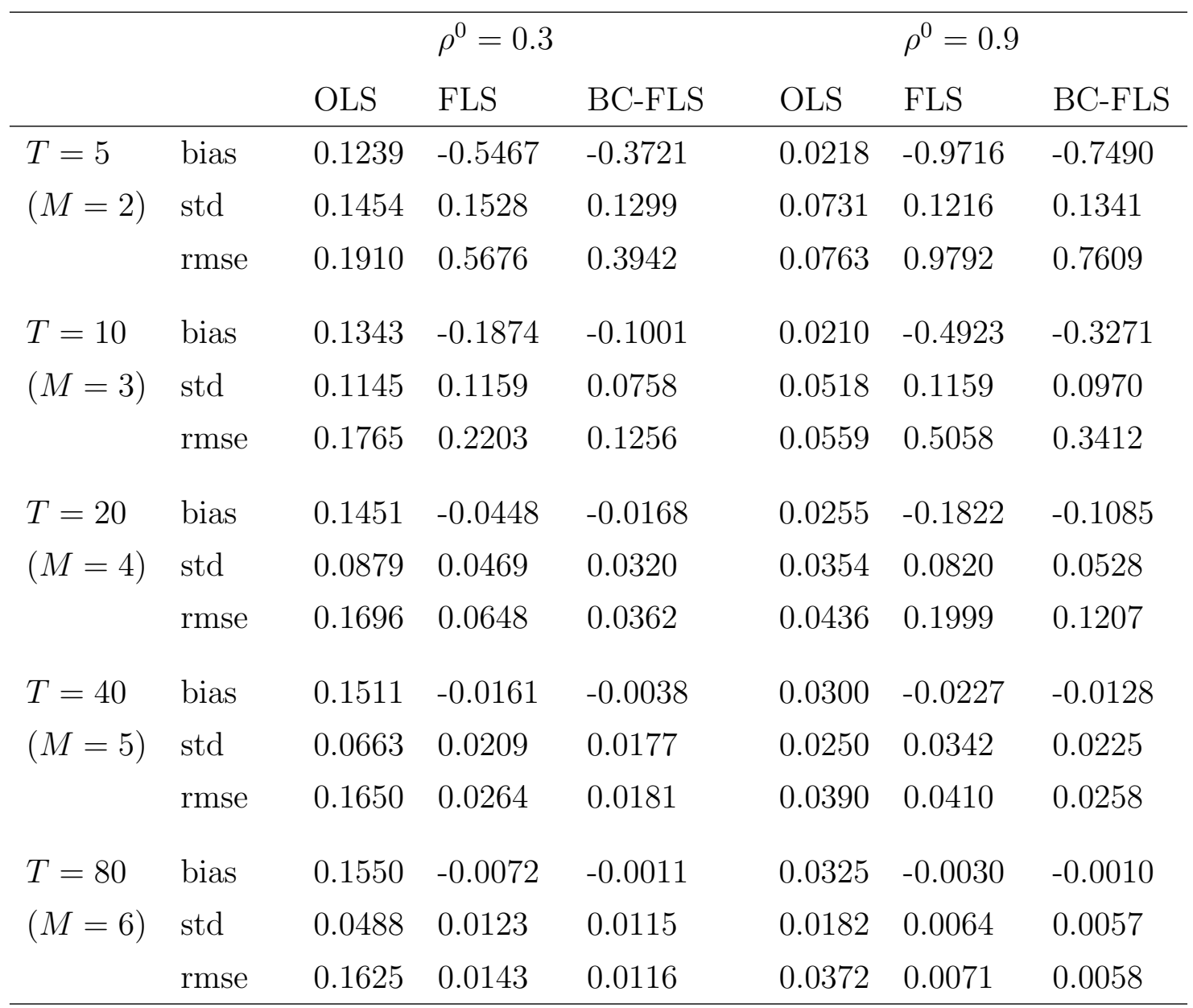

Table 3: Simulation results for the $\operatorname{AR}(1)$ model with $N=100, T=20, \rho_{f}=0.5$, and $\sigma_{f}=0.5$. For different values of the $\operatorname{AR}(1)$ coefficient $\rho^{0}$ and of the bandwidth $M$, we give the fraction of the LS estimator bias that is accounted for by the bias correction, i.e. the fraction $\sqrt{N T} \mathbb{E}(\widehat{\beta}-\beta) / \mathbb{E}\left(\widehat{W}^{-1} \widehat{B}\right)$, computed over 10,000 simulation runs. Here and in all following tables it is assumed that $R=1$ is correctly specified.

\begin{tabular}{lllllllll}
\hline & $M=1$ & $M=2$ & $M=3$ & $M=4$ & $M=5$ & $M=6$ & $M=7$ & $M=8$ \\
\hline$\rho^{0}=0$ & 0.889 & 0.832 & 0.791 & 0.754 & 0.720 & 0.689 & 0.660 & 0.633 \\
$\rho^{0}=0.3$ & 0.752 & 0.806 & 0.778 & 0.742 & 0.708 & 0.677 & 0.648 & 0.621 \\
$\rho^{0}=0.6$ & 0.589 & 0.718 & 0.728 & 0.704 & 0.674 & 0.644 & 0.616 & 0.590 \\
$\rho^{0}=0.9$ & 0.299 & 0.428 & 0.486 & 0.510 & 0.519 & 0.516 & 0.508 & 0.495 \\
\hline
\end{tabular}




\section{Dynamic Panel With Interactive Effects}

Table 4: Same specification as Table 1. We only report the properties of the bias-corrected LS estimator, but for multiple values of the bandwidth parameter $M$ and two different values for T. Results were obtained using 10,000 simulation runs.

\begin{tabular}{llllllll}
\hline & & \multicolumn{3}{c}{ BC-FLS for $\rho^{0}=0.3$} & \multicolumn{3}{c}{ BC-FLS for $\rho^{0}=0.9$} \\
& & $\mathrm{M}=2$ & $\mathrm{M}=5$ & $\mathrm{M}=8$ & $\mathrm{M}=2$ & $\mathrm{M}=5$ & $\mathrm{M}=8$ \\
\hline$T=20$ & bias & -0.0056 & -0.0082 & -0.0100 & -0.0100 & -0.0083 & -0.0089 \\
& std & 0.0239 & 0.0241 & 0.0247 & 0.0253 & 0.0212 & 0.0208 \\
& rmse & 0.0245 & 0.0255 & 0.0266 & 0.0272 & 0.0228 & 0.0227 \\
$T=40$ & bias & -0.0017 & -0.0023 & -0.0030 & -0.0024 & -0.0019 & -0.0018 \\
& std & 0.0159 & 0.0159 & 0.0159 & 0.0095 & 0.0089 & 0.0085 \\
& rmse & 0.0160 & 0.0161 & 0.0162 & 0.0098 & 0.0091 & 0.0087 \\
\hline
\end{tabular}

Table 5: Simulation results for the AR(1) model with $N=100, T=20, M=4$, and $\rho^{0}=0.6$. The three different estimators were computed for 10,000 simulation runs, and the mean bias, standard deviation (std), and root mean square error (rmse) are reported.

\begin{tabular}{llllllll}
\hline & & \multicolumn{3}{c}{$\rho_{f}=0.3$} & & \multicolumn{3}{c}{$\rho_{f}=0.7$} \\
\hline$\sigma_{f}=0$ & bias & -0.0007 & -0.0076 & -0.0043 & -0.0004 & -0.0074 & -0.0041 \\
& std & 0.0182 & 0.0332 & 0.0243 & 0.0178 & 0.0331 & 0.0242 \\
& rmse & 0.0182 & 0.0340 & 0.0247 & 0.0178 & 0.0339 & 0.0245 \\
$\sigma_{f}=0.2$ & bias & 0.0153 & -0.0113 & -0.0032 & 0.0474 & -0.0291 & -0.0071 \\
& std & 0.0251 & 0.0303 & 0.0229 & 0.0382 & 0.0387 & 0.0272 \\
& rmse & 0.0294 & 0.0323 & 0.0231 & 0.0609 & 0.0484 & 0.0281 \\
$\sigma_{f}=0.5$ & bias & 0.0567 & -0.0137 & -0.0041 & 0.1491 & -0.0403 & -0.0126 \\
& std & 0.0633 & 0.0260 & 0.0207 & 0.0763 & 0.0298 & 0.0226 \\
& rmse & 0.0850 & 0.0294 & 0.0211 & 0.1675 & 0.0501 & 0.0259 \\
\hline
\end{tabular}




\section{Dynamic Panel With Interactive Effects}

Table 6: Simulation results for the $\operatorname{AR}(1)$ model with $\rho_{f}=0.5$ and $\sigma_{f}=0.5$. For the different values of $\rho^{0}, N, T$, and $M$, we test the hypothesis $H_{0}: \rho=\rho^{0}$ using the uncorrected and biascorrected Wald, LR, and LM test, and nominal size 5\%. The bias-corrected tests are indicated by an asterisk superscript. The size of the different tests is reported, based on 10,000 simulation runs.

\begin{tabular}{lcccccc}
\hline & \multicolumn{3}{c}{ size } & \multicolumn{4}{c}{ size } \\
& $W D$ & $L R$ & $L M$ & $W D^{*}$ & $L R^{*}$ & $L M^{*}$ \\
\hline$\rho^{0}=0$ & & & & & & \\
$N=100, T=20, M=4$ & 0.219 & 0.214 & 0.192 & 0.066 & 0.062 & 0.056 \\
$N=400, T=80, M=6$ & 0.199 & 0.198 & 0.195 & 0.055 & 0.054 & 0.054 \\
$N=400, T=20, M=4$ & 0.560 & 0.556 & 0.532 & 0.089 & 0.088 & 0.076 \\
$N=1600, T=80, M=6$ & 0.593 & 0.591 & 0.586 & 0.056 & 0.055 & 0.055 \\
$\rho^{0}=0.6$ & & & & & & \\
$N=100, T=20, M=4$ & 0.326 & 0.311 & 0.272 & 0.098 & 0.091 & 0.077 \\
$N=400, T=80, M=6$ & 0.260 & 0.255 & 0.248 & 0.056 & 0.053 & 0.057 \\
$N=400, T=20, M=4$ & 0.591 & 0.582 & 0.552 & 0.174 & 0.167 & 0.136 \\
$N=1600, T=80, M=6$ & 0.666 & 0.663 & 0.656 & 0.060 & 0.058 & 0.059 \\
\hline
\end{tabular}




\section{Dynamic Panel With Interactive Effects}

Table 7: As Table 6, but we report the power for testing the alternatives $H_{a}^{\text {left }}: \rho=\rho^{0}-(N T)^{-1 / 2}$ and $H_{a}^{\text {right }}: \rho=\rho^{0}+(N T)^{-1 / 2}$. The bias-corrected tests are indicated by an asterisk superscript.

\begin{tabular}{|c|c|c|c|c|c|c|c|}
\hline & \multicolumn{3}{|c|}{ power } & \multicolumn{3}{|c|}{ power } \\
\hline & & $W D$ & $L R$ & $L M$ & $W D^{*}$ & $L R^{*}$ & $L M^{*}$ \\
\hline \multicolumn{8}{|l|}{$\rho^{0}=0$} \\
\hline \multirow[t]{2}{*}{$N=100, T=20, M=4$} & $H_{a}^{\text {left }}$ & 0.094 & 0.089 & 0.076 & 0.128 & 0.123 & 0.121 \\
\hline & $H_{a}^{\text {right }}$ & 0.526 & 0.515 & 0.487 & 0.235 & 0.227 & 0.206 \\
\hline \multirow[t]{2}{*}{$N=400, T=80, M=6$} & $H_{a}^{\text {left }}$ & 0.066 & 0.064 & 0.063 & 0.154 & 0.151 & 0.153 \\
\hline & $H_{a}^{\text {right }}$ & 0.549 & 0.545 & 0.540 & 0.194 & 0.191 & 0.190 \\
\hline \multirow[t]{2}{*}{$N=400, T=20, M=4$} & $H_{a}^{\text {left }}$ & 0.306 & 0.305 & 0.284 & 0.100 & 0.097 & 0.096 \\
\hline & $H_{a}^{\text {right }}$ & 0.791 & 0.787 & 0.769 & 0.309 & 0.305 & 0.279 \\
\hline \multirow{2}{*}{$N=1600, T=80, M=6$} & $H_{a}^{\text {left }}$ & 0.254 & 0.253 & 0.248 & 0.128 & 0.127 & 0.129 \\
\hline & $H_{a}^{\text {right }}$ & 0.871 & 0.869 & 0.866 & 0.225 & 0.224 & 0.224 \\
\hline \multicolumn{8}{|l|}{$\rho^{0}=0.6$} \\
\hline \multirow[t]{2}{*}{$N=100, T=20, M=4$} & $H_{a}^{\text {left }}$ & 0.192 & 0.180 & 0.147 & 0.184 & 0.171 & 0.171 \\
\hline & $H_{a}^{\text {right }}$ & 0.619 & 0.605 & 0.563 & 0.335 & 0.318 & 0.294 \\
\hline \multirow[t]{2}{*}{$N=400, T=80, M=6$} & $H_{a}^{\text {left }}$ & 0.081 & 0.079 & 0.076 & 0.184 & 0.195 & 0.200 \\
\hline & $H_{a}^{\text {right }}$ & 0.680 & 0.675 & 0.668 & 0.335 & 0.262 & 0.267 \\
\hline \multirow[t]{2}{*}{$N=400, T=20, M=4$} & $H_{a}^{\text {left }}$ & 0.421 & 0.412 & 0.378 & 0.184 & 0.160 & 0.150 \\
\hline & $H_{a}^{\text {right }}$ & 0.792 & 0.787 & 0.765 & 0.335 & 0.426 & 0.399 \\
\hline \multirow[t]{2}{*}{$N=1600, T=80, M=6$} & $H_{a}^{\text {left }}$ & 0.318 & 0.314 & 0.307 & 0.200 & 0.169 & 0.172 \\
\hline & $H_{a}^{\text {right }}$ & 0.912 & 0.911 & 0.908 & 0.268 & 0.316 & 0.320 \\
\hline
\end{tabular}




\section{Dynamic Panel With Interactive Effects}

Table 8: As Table 7, but we report the size-corrected power.

\begin{tabular}{|c|c|c|c|c|c|c|c|}
\hline & & \multicolumn{3}{|c|}{ size-corrected power } & \multicolumn{3}{|c|}{ size-corrected power } \\
\hline & & $W D$ & $L R$ & $L M$ & $W D^{*}$ & $L R^{*}$ & $L M^{*}$ \\
\hline \multicolumn{8}{|l|}{$\rho^{0}=0$} \\
\hline \multirow[t]{2}{*}{$N=100, T=20, M=4$} & $H_{a}^{\text {left }}$ & 0.010 & 0.011 & 0.010 & 0.105 & 0.104 & 0.112 \\
\hline & $H_{a}^{\text {right }}$ & 0.211 & 0.208 & 0.206 & 0.199 & 0.197 & 0.193 \\
\hline \multirow[t]{2}{*}{$N=400, T=80, M=6$} & $H_{a}^{\text {left }}$ & 0.008 & 0.008 & 0.008 & 0.143 & 0.143 & 0.145 \\
\hline & $H_{a}^{\text {right }}$ & 0.236 & 0.237 & 0.235 & 0.181 & 0.182 & 0.181 \\
\hline \multirow{2}{*}{$N=400, T=20, M=4$} & $H_{a}^{\text {left }}$ & 0.008 & 0.008 & 0.009 & 0.055 & 0.052 & 0.062 \\
\hline & $H_{a}^{\text {right }}$ & 0.187 & 0.185 & 0.181 & 0.210 & 0.208 & 0.208 \\
\hline \multirow[t]{2}{*}{$N=1600, T=80, M=6$} & $H_{a}^{\text {left }}$ & 0.005 & 0.005 & 0.005 & 0.119 & 0.119 & 0.120 \\
\hline & $H_{a}^{\text {right }}$ & 0.226 & 0.227 & 0.225 & 0.213 & 0.213 & 0.212 \\
\hline \multicolumn{8}{|l|}{$\rho^{0}=0.6$} \\
\hline \multirow[t]{2}{*}{$N=100, T=20, M=4$} & $H_{a}^{\text {left }}$ & 0.014 & 0.014 & 0.016 & 0.114 & 0.115 & 0.127 \\
\hline & $H_{a}^{\text {right }}$ & 0.196 & 0.193 & 0.196 & 0.233 & 0.234 & 0.231 \\
\hline \multirow[t]{2}{*}{$N=400, T=80, M=6$} & $H_{a}^{\text {left }}$ & 0.005 & 0.005 & 0.005 & 0.114 & 0.187 & 0.184 \\
\hline & $H_{a}^{\text {right }}$ & 0.288 & 0.288 & 0.288 & 0.233 & 0.252 & 0.247 \\
\hline \multirow[t]{2}{*}{$N=400, T=20, M=4$} & $H_{a}^{\text {left }}$ & 0.013 & 0.016 & 0.015 & 0.114 & 0.039 & 0.051 \\
\hline & $H_{a}^{\text {right }}$ & 0.128 & 0.127 & 0.126 & 0.233 & 0.201 & 0.209 \\
\hline \multirow[t]{2}{*}{$N=1600, T=80, M=6$} & $H_{a}^{\text {left }}$ & 0.005 & 0.005 & 0.005 & 0.185 & 0.153 & 0.154 \\
\hline & $H_{a}^{\text {right }}$ & 0.236 & 0.236 & 0.238 & 0.248 & 0.291 & 0.291 \\
\hline
\end{tabular}

